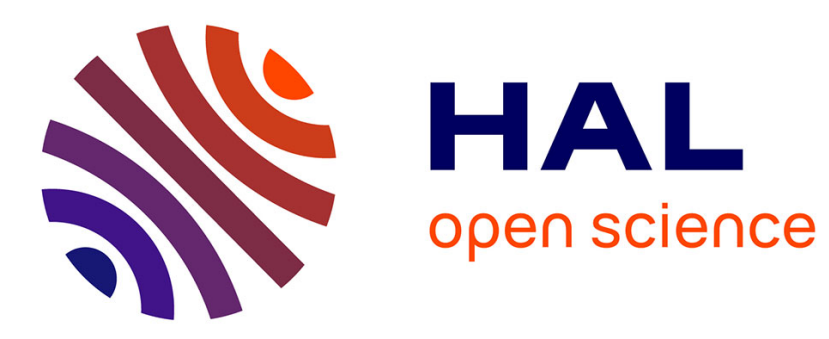

\title{
Un matérialisme affectif
}

Pierre-Olivier Dittmar, Pierre Antoine Fabre, Thomas Golsenne, Caroline

Perrée

\section{To cite this version:}

Pierre-Olivier Dittmar, Pierre Antoine Fabre, Thomas Golsenne, Caroline Perrée. Un matérialisme affectif. Techniques et culture, 2018, Matérialiser les désirs. Techniques votives, 70 (2), pp.12 - 41. $10.4000 /$ tc. 9382 . halshs- 03221240

\section{HAL Id: halshs-03221240 \\ https://shs.hal.science/halshs-03221240}

Submitted on 11 May 2021

HAL is a multi-disciplinary open access archive for the deposit and dissemination of scientific research documents, whether they are published or not. The documents may come from teaching and research institutions in France or abroad, or from public or private research centers.
L'archive ouverte pluridisciplinaire HAL, est destinée au dépôt et à la diffusion de documents scientifiques de niveau recherche, publiés ou non, émanant des établissements d'enseignement et de recherche français ou étrangers, des laboratoires publics ou privés. 


\section{UN MATÉRIALISME AFFECTIF}

\section{Pierre-Olivier Dittmar, Pierre-Antoine Fabre, Thomas Golsenne, Caroline Perrée}

\section{Éditions de l'EHESS | «Techniques \& Culture »}

$2018 / 2 \mathrm{n}^{\circ} 70 \mid$ pages 12 à 41

ISSN 0248-6016

Article disponible en ligne à l'adresse :

https://www.cairn.info/revue-techniques-et-culture-2018-2-page-12.htm

Distribution électronique Cairn.info pour Éditions de l'EHESS.

(c) Éditions de l'EHESS. Tous droits réservés pour tous pays.

La reproduction ou représentation de cet article, notamment par photocopie, n'est autorisée que dans les limites des conditions générales d'utilisation du site ou, le cas échéant, des conditions générales de la licence souscrite par votre établissement. Toute autre reproduction ou représentation, en tout ou partie, sous quelque forme et de quelque manière que ce soit, est interdite sauf accord préalable et écrit de l'éditeur, en dehors des cas prévus par la législation en vigueur en France. Il est précisé que son stockage dans une base de données est également interdit. 


\section{Techniques \& Culture}

Revue semestrielle d'anthropologie des techniques

$70 \mid 2018$

Matérialiser les désirs

\section{Un matérialisme affectif}

Pierre-Olivier Dittmar, Pierre Antoine Fabre, Thomas Golsenne et Caroline Perrée

(2) OpenEdition

Journals

Édition électronique

URL : http://journals.openedition.org/tc/9382

DOI : $10.4000 /$ tc. 9382

ISSN : 1952-420X

Éditeur

Éditions de l'EHESS

Édition imprimée

Date de publication : 30 octobre 2018

Pagination : $12-41$

ISBN : 2-7132-2751-6

ISSN : 0248-6016

Distribution électronique Cairn

\section{CAIRN INFO}

CHERCHER, REPÉRER, AVANCER

Référence électronique

Pierre-Olivier Dittmar, Pierre Antoine Fabre, Thomas Golsenne et Caroline Perrée, « Un matérialisme affectif », Techniques \& Culture [En ligne], 70 | 2018, mis en ligne le 06 décembre 2020, consulté le 16 janvier 2019. URL : http://journals.openedition.org/tc/9382 ; DOI : 10.4000/tc.9382 


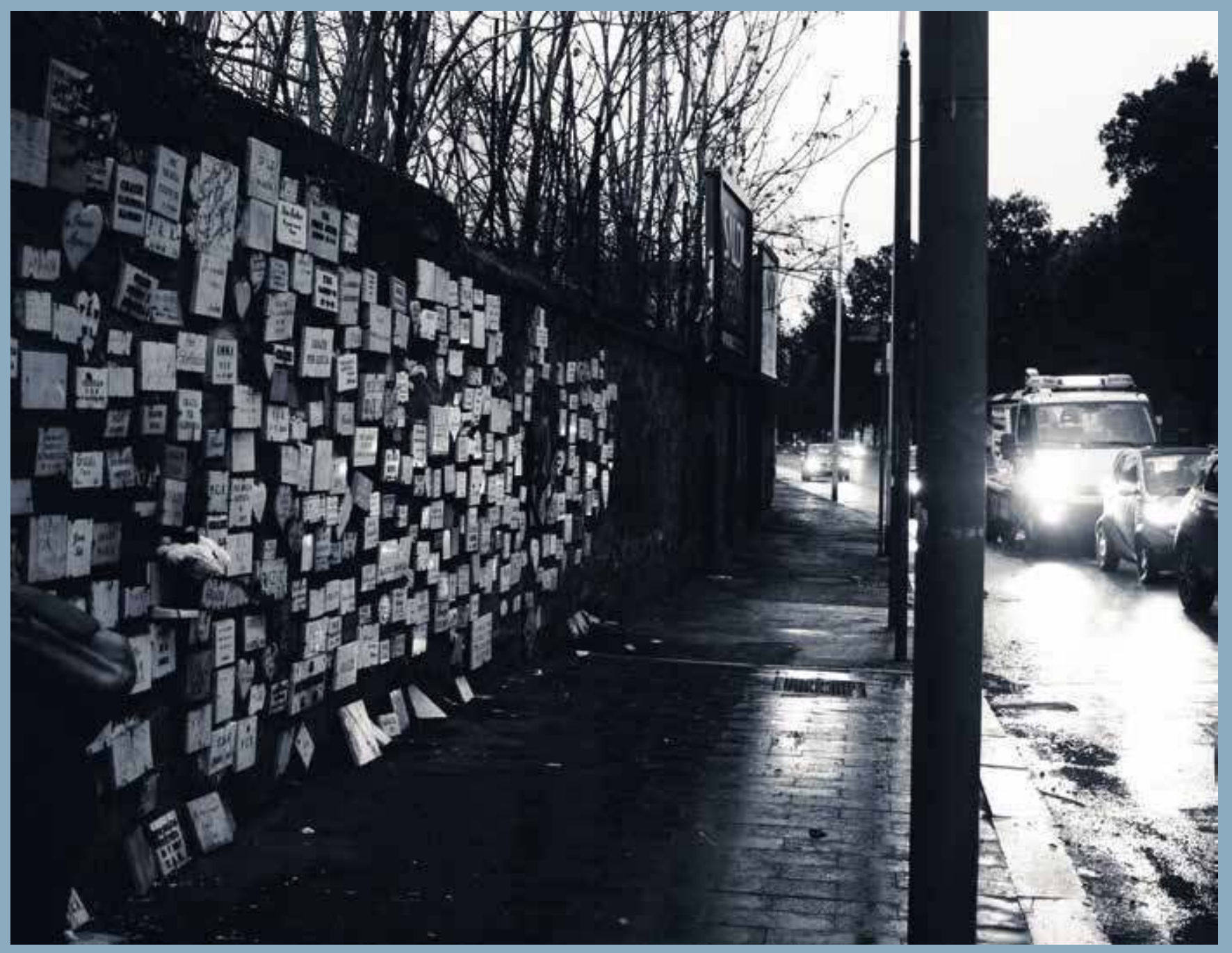




\title{
Un matérialisme affectif
}

\begin{abstract}
«Diagoras, celui que l'on appelle l'Athée, étant à Samothrace, un de ses amis lui demanda: "Toi qui penses que les dieux négligent les affaires humaines, ne vois-tu pas, d'après tous ces tableaux peints, que nombreux furent ceux qui échappèrent à la force de la tempête par leurs vœux et parvinrent sains et saufs au port?" Et lui de répondre: "C'est vrai. Mais je ne vois pas où sont peints ceux qui firent naufrage et périrent en mer." ${ }^{1}$. Cicéron, De la nature des dieux, Livre III, 37, 89.
\end{abstract}

Pour qui, un soir, irait marcher dans la banlieue de Rome, le long du Largo Preneste, axe routier sans charme, deux fois trois voies bordées de vieilles industries, pour cette personne-là, à supposer qu'elle soit attentive, scintilleraient dans la lueur des phares, le long d'un mur de briques, des centaines de plaques de marbres fixées fragilement. Plus près, elle verrait des inscriptions énigmatiques, plusieurs fois «P.R.G.», des initiales, et des demandes, des remerciements de toutes sortes, désirs d'amour, de santé, de travail. S'attardant encore un peu, elle remarquerait, glissés entre les briques, des petits animaux en plastique, des cœurs rouillés, des lettres entières rédigées au crayon à papier, glissées derrière le marbre. Des photos. Des couples, des accidents. Des menottes mal scellées par du plâtre.

Loin de toute église, loin des lieux de pèlerinage touristique, cet angle de rue témoigne, exemple entre mille, de la pratique obstinée, au sein de nos sociétés contemporaines, d'exprimer ses désirs et sa gratitude avec un peu de matière.

\section{Extension du domaine de l'ex-voto}

Qu'est-ce que l'on dit avec quelque «chose» que l'on ne pourrait dire avec des mots? La forme ex-voto offre un paradigme de première envergure pour penser la place de la matérialité dans 
les pratiques d'échange avec les invisibles: cherche-t-on à passer du côté de l'invisible et de ses puissances par des objets transitionnels ou à ferrer ces puissances dans le visible par ces appâts? Des appâts qui étendent la définition de la «chose » et de l'« objet » jusqu'à l'infini -ou jusqu'à l'humanité elle-même, comme dans le cas des enfants donnés comme oblats, c'est-àdire comme offrandes vivantes, aux monastères médiévaux en remerciement d'une grâce reçue; ou encore lorsque l'on donne une partie de soi, ou son corps entier quand on lui impose des gestes, des transformations, etc.

Depuis la Chypre du néolithique, dans la Grande Grèce du ve siècle, dans l'Italie du Xve siècle, sur les îles Trobriand jusqu'au siècle dernier, à Paris ou à Tokyo au xxie siècle, des femmes et des hommes expriment leurs souffrances et leurs désirs par de tels «objets». Cette documentation remarquable a trop souvent été cantonnée aux inventaires et aux études sur les arts et dévotions populaires. Elle ne rend pas compte de la diversité des acteurs et des affects qui ont historiquement porté ces pratiques, ni des formidables transformations du phénomène votif au cours de ces vingt dernières années, à l'échelle de la planète. Il n'est plus possible aujourd'hui de considérer ces conduites comme les ultimes feux d'une pratique ancestrale: les dernières décennies ont vu se multiplier les dépôts spontanés dans l'espace public d'objets, de choses, qui chacun à leur façon exprimait une demande ou un remerciement, des affects. Si l'accumulation d'organes malades, de béquilles ou de plâtres force à penser l'exposition collective de souffrances intimes, la diversité des pratiques contemporaines oblige à re-situer le phénomène dans le cadre d'une société de consommation et invite à tenir compte des photographies de motos ou autres engins désirables, des T-shirts de footballeurs, des serments d'amour et des manifestations de gratitude pour un diplôme ou pour un permis de conduire, autant de pratiques qui incitent à déborder la notion de vœu (paien puis chrétien) pour trouver, celle plus englobante, de désir.

Les cadenas d'amour verrouillés aux ponts qui se multiplient dans le monde urbain depuis les années quatre-vingt-dix ne sont qu'un exemple, parmi d'autres, d'un matérialisme affectif qui, parfois sans divinité et même sans lieu de culte, s'impose comme une modalité contemporaine du rapport à l'espace public.

On ne peut plus désormais se limiter au monde des maquettes de bateaux et des petites peintures «naïves». L'image n'est plus le modèle par excellence de l'ex-voto et les pouvoirs de la ressemblance ne sont plus les seuls opératoires pour comprendre son efficacité. Il faut rendre à la pratique toute sa diversité: amas de cire disposés dans les églises, fruits et légumes déposés devant des autels, figurines animales, mais aussi tableaux de Raphaël, de Poussin ou d'Ingres, places de villes ou églises, inscriptions gravées dans le marbre ou écrites à la main, objets industriels et personnes vivantes, tout ou presque peut être investi d'une intention votive.

Mais s'agit-il d'un élargissement profane et contemporain de la sphère de l'ex-voto, ou de nouvelles catégories d'objets qui n'ont que peu à voir avec les cires anatomiques et les récits de miracles peints sur des tableaux? Il nous semble qu'une démarche d'anthropologie comparative et historique permet de répondre à cette question et de faire apparaître dans les pratiques anciennes des dimensions qui leur étaient restées souterraines, en particulier pour ce qui concerne leurs enjeux sociaux, collectifs, communautaires. 
Dans la dernière décennie, la recherche a été largement renouvelée par une série d'enquêtes des deux côtés de l'Atlantique. En Amérique latine tout d'abord, avec le projet Ex voto das americas, aux États-Unis, grâce à une série de publications et une exposition au Bard Center, à New York, Agents of Faith: Material, Place, Memory, qui a lieu tandis que ces lignes sont imprimées, en France enfin, où un séminaire ouvert depuis trois ans à l'EHESS ("Saintetés, sacralités, dévotions. Pour une anthropologie historique de l'objet votif ») a contribué à redéfinir les cadres scientifiques d'une interrogation sur le sens des objets et des pratiques votives ${ }^{2}$.

On le comprend, il s'agit dans ce numéro de Techniques ECulture d'approcher la chose et le problème par ses limites, en embrassant dans un même mouvement, d'une part l'extraordinaire stabilité de formes relevée par Georges Didi-Huberman (2006), qui impose de penser la présence d'objets quasiment similaires dans les dépôts votifs celtiques et les sanctuaires chrétiens du xxi siècle, et d'autre part un besoin d'histoire toujours plus urgent, face à un phénomène totalement plastique, dont la compréhension est constamment mobile.

Si l'ex-voto a fasciné, dans sa diversité, ses permanences et son inventivité, il a peut-être aussi paralysé historiens et historiens de l'art dans un face-à-face avec l'objet qui les empêchait de saisir toute une série d'enjeux sociaux, affectifs et politiques que la pratique elle-même engageait. C'est pourquoi nous avons voulu, autant que possible, mettre à distance l'ex-voto pour considérer des techniques votives, comme différents moyens d'associer des intentions et de la matière. Il s'agit dès lors de ne pas considérer les ex-voto comme des objets clos, mais comme des choses en perpétuel devenir. Cela concerne bien sûr les procédés de fabrication, les chaînes opératoires visant soit à produire un objet spécifique pour un vœu, soit à transformer des objets courants en porteurs d'intention. Cela concerne surtout le moment même, souvent transitoire, où l'objet votif existe en tant qu'objet votif, le temps de sa visibilité. Ici, nous avons voulu porter une attention particulière aux dispositifs, au sens foucaldien, c'est-à-dire à la façon dont des lieux, des espaces, des ornements, mais aussi des textes, des récits, pouvaient s'articuler pour conférer une forme d'efficacité à ces objets. S'ils visent à produire des effets, ces agencements témoignent aussi de rapports de force chaque fois singuliers, d'arbitrages particulièrement sensibles entre des corps individuels et le corps social, entre des politiques institutionnelles et des souffrances individuelles, qui ne s'accordent pas toujours. 
Enfin, il nous a semblé indispensable de penser l'acte votif au-delà de la matérialité qu'il impliquait pour considérer les actions rituelles, les gestes des femmes et des hommes qui ont déposé ces objets, les ont façonnés, etc.

À partir de l'Europe et de l'Amérique chrétiennes, qui réunissaient l'essentiel de nos domaines de compétence, nous avons collectivement élaboré, dans le cadre du séminaire de l'EHESS et de la préparation de ce numéro, une définition propre à rendre possible la plus large comparabilité (ou à découvrir des incomparables). La voici: L'ex-voto est un don physique à une puissance supposée agissante en un lieu spécifique et l'expression d'un désir formulé ou assouvi. Ce don est l'acte d'un individu ou d'un groupe, toujours environné d'autres dons du même type.

\section{Les nouveaux invisibles}

Si nous avons voulu notre définition la plus englobante possible, permet-elle de rendre compte de la diversité et de la multiplication des pratiques contemporaines? Tandis que les dons votifs ne font plus l'objet de commandes de prestige par les princes, il faut constater avec Ceri Houlbrook que loin de disparaître, les «agencements populaires » d'objets les plus variés, arbres à chaussures, cadenas d'amour, cairns, dépôts mémoriels après un accident ou un attentat, se multiplient depuis les années 1990. S'il existe une continuité des gestes, des pratiques, ces agencements anonymes peuvent se faire sans référence à une divinité, et laisser émerger de «nouveaux invisibles». Que se passe-t-il quand un touriste dépose, au pied d'une copie de la flamme de la statue de la Liberté du pont de l'Alma, à Paris, un remerciement à la princesse Diana pour «son petit appartement» (voir le portfolio préparé par Émilie Girard avec les collections du Mucem)? Pourquoi, quelques années plus tard, le même lieu accueille-t-il des cadenas d'amour? Le mur de la maison de «Juliette» à Vérone est aujourd'hui recouvert par une étrange composition collective, des lettres d'amour, demandes ou remerciements, collées par des morceaux de chewing-gums sur les pierres vénérables du domicile supposé de l'héroïne tragique. Par quelles voies une princesse morte dans un accident de la route, une héroïne littéraire suicidée deviennent-elles des intercesseuses sollicitées dans des domaines aussi divers que la recherche de l'âme sœur ou celle d'un appartement?

Force est de constater qu'il existe une diffusion horizontale des désirs par les «choses», qui ne répond plus au schéma d'une transcendance. Lorsque l'on accroche un cadenas sur un autre cadenas, lorsque l'on glisse une lettre de demande derrière un vieil ex-voto plutôt que devant une image de culte, toutes ces techniques de détournement et de réemploi contribuent à faire de chacun un acteur et donc, d'une certaine manière, un intercesseur, alors que, dans les cas où la pratique des ex-voto est encadrée par une structure religieuse, l'intercesseur est la puissance incarnée qui investit le lieu sacré. Ce phénomène oblige ainsi à considérer les situations les plus radicales, auxquelles s'attachent de «nouveaux invisibles » : la maison de Juliette ne peut se comprendre sans évoquer les gum walls qui se multiplient autour du globe, pans de 
mur recouverts de chewing-gums mâchés, ou encore le désormais disparu ponte delle gomme de Venise, où les amoureux des années 2000 venaient coller de leurs salives mélangées un souvenir (ou, parfois, un vœu).

À ce titre, les remarquables coin trees des îles britanniques sont particulièrement riches d'enseignement puisque Ceri Houlbrook montre que ce qui était autrefois une pratique votive attestée (insérer des pièces de monnaie dans l'écorce d'un arbre) ne cesse de prendre de l'ampleur quand bien même aucun des acteurs rencontrés dans son enquête n'affirme avoir formulé un vœu.

Sans divinités, sans vœux, ces agencements

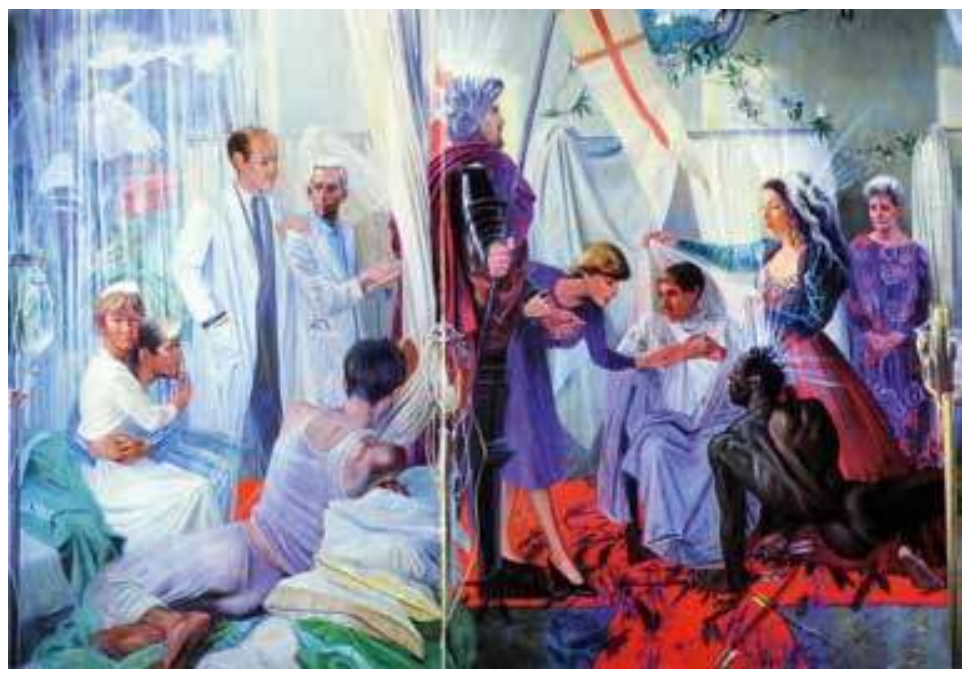
nous obligent à considérer à nu un désir toujours plus fort de faire éclore des communautés d'actants anonymes avec la complicité d'un monde matériel réinventé. Ce collectif, plus que l'institution en charge du lieu, souvent réfractaire à la matérialité de ces pratiques, valide, en la perpétuant, l'efficacité de la topographie votive (sur un mode parodique même, ce que remarque Anne Lepoittevin dans son étude du Pasquino romain). L'ex-voto, ainsi conçu, devient un objet témoin, au service du rayonnement et de la puissance du sanctuaire aux yeux des autres pèlerins, comme le note Agnès Giard au Japon.

De prime abord chaotique, l'accrochage sauvage - dans les sanctuaires anciens comme dans les «temples» sans dieux ni vœux-dessine peu à peu une scénographie du don, mimétiquement reprise par l'ensemble des pèlerins. Il peut alors échapper aux autorités et aux limites construites du sanctuaire pour sacraliser des éléments naturels comme les arbres (reprenant parfois d'anciens rituels, comme l'observe Vasiliki Zachari en Grèce). Au sanctuaire d'Istanbul, ces derniers deviennent le support de fils votifs ou de berceaux bricolés suspendus, expressions d'un désir d'enfant. À Chalma au Mexique, à quelques pas du sanctuaire qui comporte pourtant une salle consacrée aux ex-voto, les arbres reçoivent le cordon ombilical des enfants enfin nés.

En centrant notre définition sur le don, nous inscrivons l'ex-voto au registre des grands thèmes de l'anthropologie. Il faudra tirer parti de la vaste historiographie de l'anthropologie du don pour comprendre que la pratique votive ne relève pas de l'échange marchand, mais qu'elle est tout autant en opposition avec une interprétation théologique du don comme manifestation d'un «pur amour» (Le Brun 2002) ${ }^{3}$. De plus, l'ex-voto ne doit pas être confondu avec le sacrifice, même s'il en partage certains traits, ni ne peut être assimilé à toute offrande religieuse. L'ex-voto n'est pas seulement un don, c'est un don public accompagné d'une promesse, d'un vœu, d'un désir. Pourquoi est-il plus efficace d'accompagner la formulation de ce désir par un don matériel? C'est la question qui traverse tout ce numéro. 


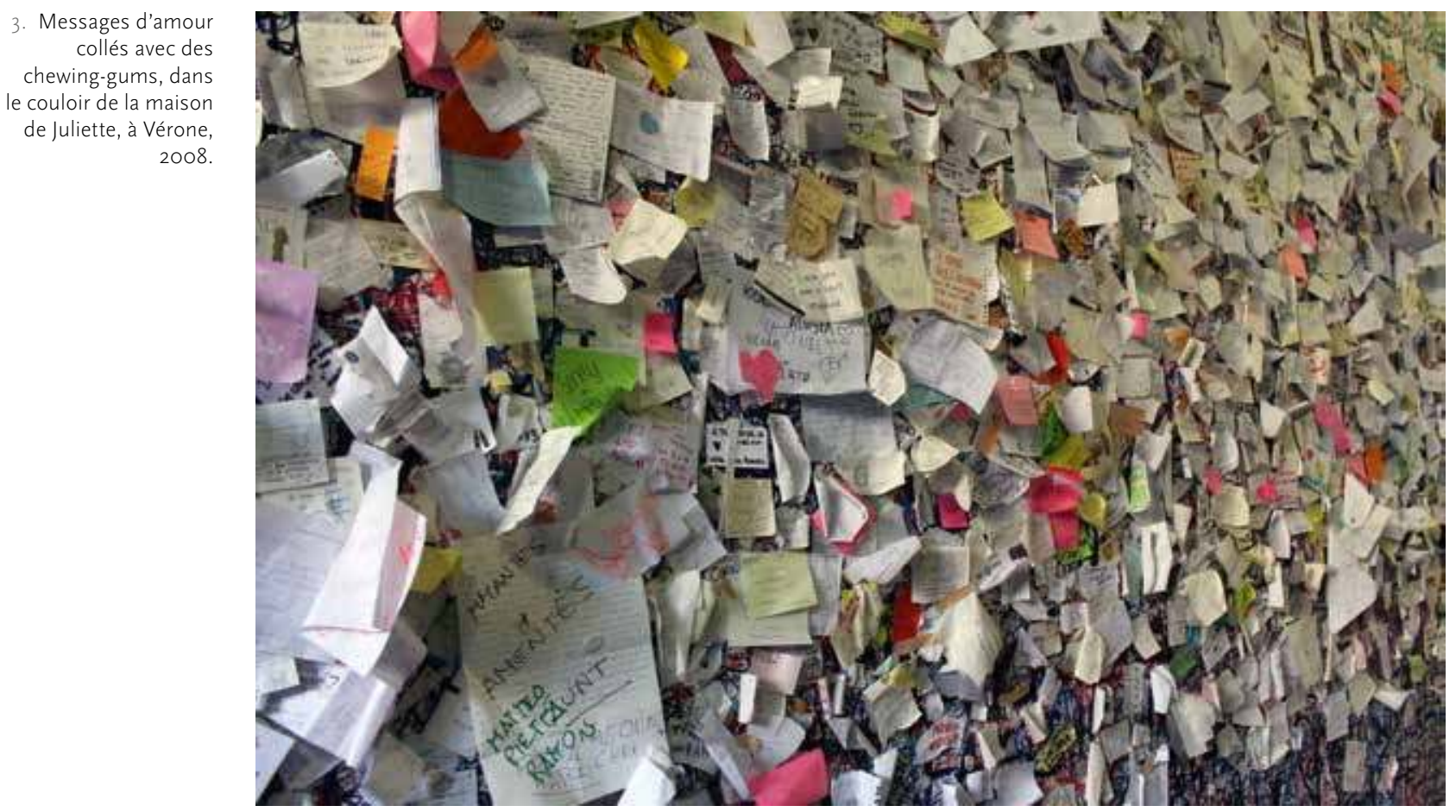

\section{I'ex-voto est-il un don?}

Le 28 mars 1486, le conseil communal d'Ascoli Piceno dans la Marche d'Ancône décide, en pleine épidémie de peste, d'édifier une chapelle votive, adossée à l'église de la SS. Annunziata, en souvenir du bienheureux Jacques de la Marche, un franciscain de la région mort en odeur de sainteté. Sur son autel, le conseil décide de mettre une image en pierre: on doit y voir la Vierge Marie tournée vers le bienheureux Jacques, qui lui tend pour la lui offrir une maquette de la ville $^{4}$ (Fabiani 1950). L'image représente l'offrande d'un ex-voto communal à la Vierge. De telles maquettes urbaines votives étaient réellement offertes à la Vierge, à Jésus ou aux saints, aux $\mathrm{XV}^{e}-\mathrm{XVI}^{\mathrm{e}}$ siècles, comme le montre Nicolas Sarzeaud dans son article. Mais c'est moins la nature de l'objet qui importe ici que l'acte de donation. Un ex-voto est toujours le résultat d'un don.

Quel besoin d'associer à une prière, à un vœu adressé à la Vierge, à Bouddha, à l'esprit d'un ancêtre, un geste-objet, un don? Les anthropologues le savent bien, l'acte du don n'est pas facile à définir. Les difficultés redoublent quand on s'aperçoit que l'ex-voto ne figure pas parmi les exemples traités par Marcel Mauss dans son célèbre Essai sur le don ${ }^{5}$, ni dans les classiques de la bibliographie ethnologique sur le don qui l'ont suivi ${ }^{6}$. En retour, les spécialistes des ex-voto ignorent la littérature sur l'anthropologie du don et hésitent sur leur conception de l'offrande votive. Tantôt l'ex-voto s'apparente à la signature d'un contrat tacite entre l'humain donataire et 
le divin récipiendaire, selon un échange de bons procédés; tantôt il s'agit surtout de manifester un vœu, réalisé ou non, et non pas une forme d'échange (Boullet 1978, Cousin 1981, Sigal 1985, Deyts 1992, Weinryb 2016) ${ }^{7}$.

Les critiques de la définition de l'ex-voto comme don sont en général des critiques de la théorie maussienne du système du don et du contre-don, du don comme échange. Ces critiques considèrent que cette théorie s'assimile à une théorie de la transaction et de la réciprocité. Si tel était le cas, rien ne différencierait le don de l'échange marchand. Or la théorie de Mauss se fonde sur la nécessité de distinguer ces deux formes de transmission. L'erreur que l'on commet habituellement, en les confondant, vient du fait qu'il est difficile d'imaginer le don comme autre chose que comme un acte gratuit et désintéressé. C'est un effet de la société moderne, dans laquelle les relations marchandes ont quasiment envahi l'ensemble des relations sociales: dans cette société, les pratiques gratuites apparaissent comme des poches de résistance ou des archaïsmes. Toutefois Mauss montre que des sociétés non dominées par les échanges marchands, des sociétés où le don est la forme d'échange dominante, ne considèrent pas le don comme un acte désintéressé et gratuit; mais elles ne l'assimilent pas non plus à une transaction commerciale et intéressée. Le don est un mélange, un acte spontané et ritualisé, une manifestation de générosité et d'intérêt, un échange de biens pour créer des liens. Dans l'acte de donner, explique Mauss, « on donne quelque chose de soi » (Mauss 1950 [1923-1924] : 161).

On pourrait, à ce stade, utiliser la théorie de Mauss pour expliquer que l'ex-voto est un don, dans lequel une personne met une part de soi, par exemple sous la forme d'un substitut anatomique, ou même se donne dans le cas de l'oblation ou du pèlerinage, et qui appelle en retour le contre-don divin. C'est le cas quand le donataire accompagne son vœu d'une offrande avant que celui-ci ne soit exaucé.

Cependant, la théorie de Mauss pose que donner, c'est manifester sa puissance. En effet, dans les rituels que l'anthropologue étudie (le fameux potlatch des Indiens de la côte nord-ouest en Amérique), les échanges de dons se produisent dans le cadre d'une compétition entre chefs de tribus; celui qui accomplit la plus grande dépense, qui donne le plus à l'autre, fait de celui-ci son obligé, qui se doit de rendre plus, sous peine de s'avouer inférieur. Mais les offrandes faites aux puissances surnaturelles ne peuvent pas s'expliquer par ces pratiques de compétition. Dans le christianisme, par exemple, le don de Dieu, la grâce, est incommensurable face aux dons humains. "Per grazia ricevuta», indiquent de nombreux ex-voto italiens: il faut comprendre le don votif comme un contre-don, qui fait suite au don divin. Ou plutôt, si l'on suit Alain Testart, comme le remboursement d'une dette (1993: 81-82). Selon l'anthropologue, qui examine les pratiques d'offrandes aux êtres surnaturels dans différentes sociétés, la dette est ce que doit une personne «inférieure » à qui une personne «supérieure » a fait un prêt, et celle-ci est en droit d'en exiger le remboursement. Certains exempla médiévaux ou légendes mexicaines modernes en donnent des exemples: untel promet de faire un don si tel saint réalise son vœu; le saint le réalise, mais untel n'accomplit pas sa promesse; il est puni par le saint: il n’a pas remboursé sa dette.
Légendes des figures suivantes:

4. Vœux noués, tissus et emballages plastiques

Château de Saint-Hilarion, Chypre, 12 juillet 2017

5. Arbre à clous

Gravenvoeren, Voeren, Limburg, Belgium 


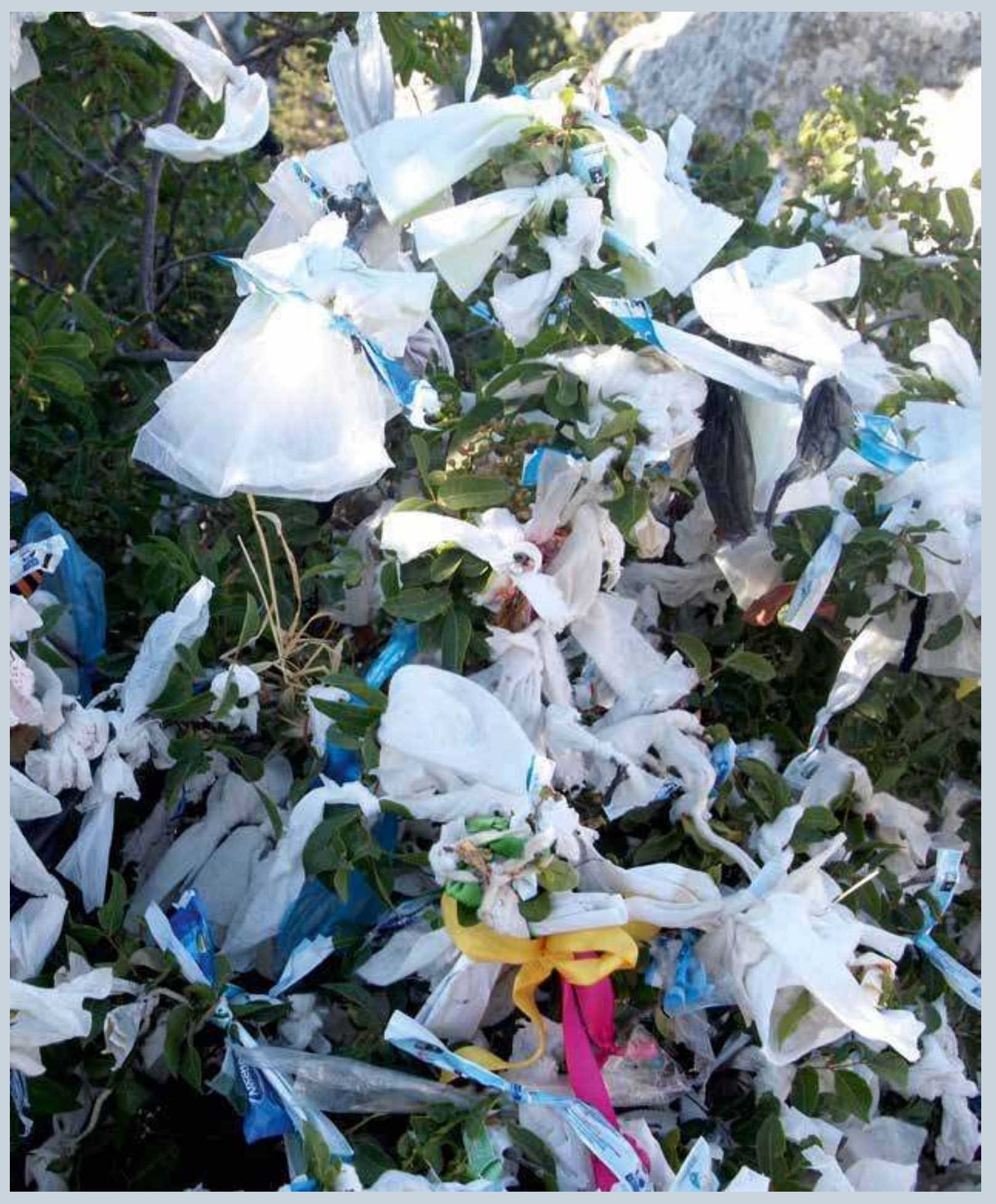




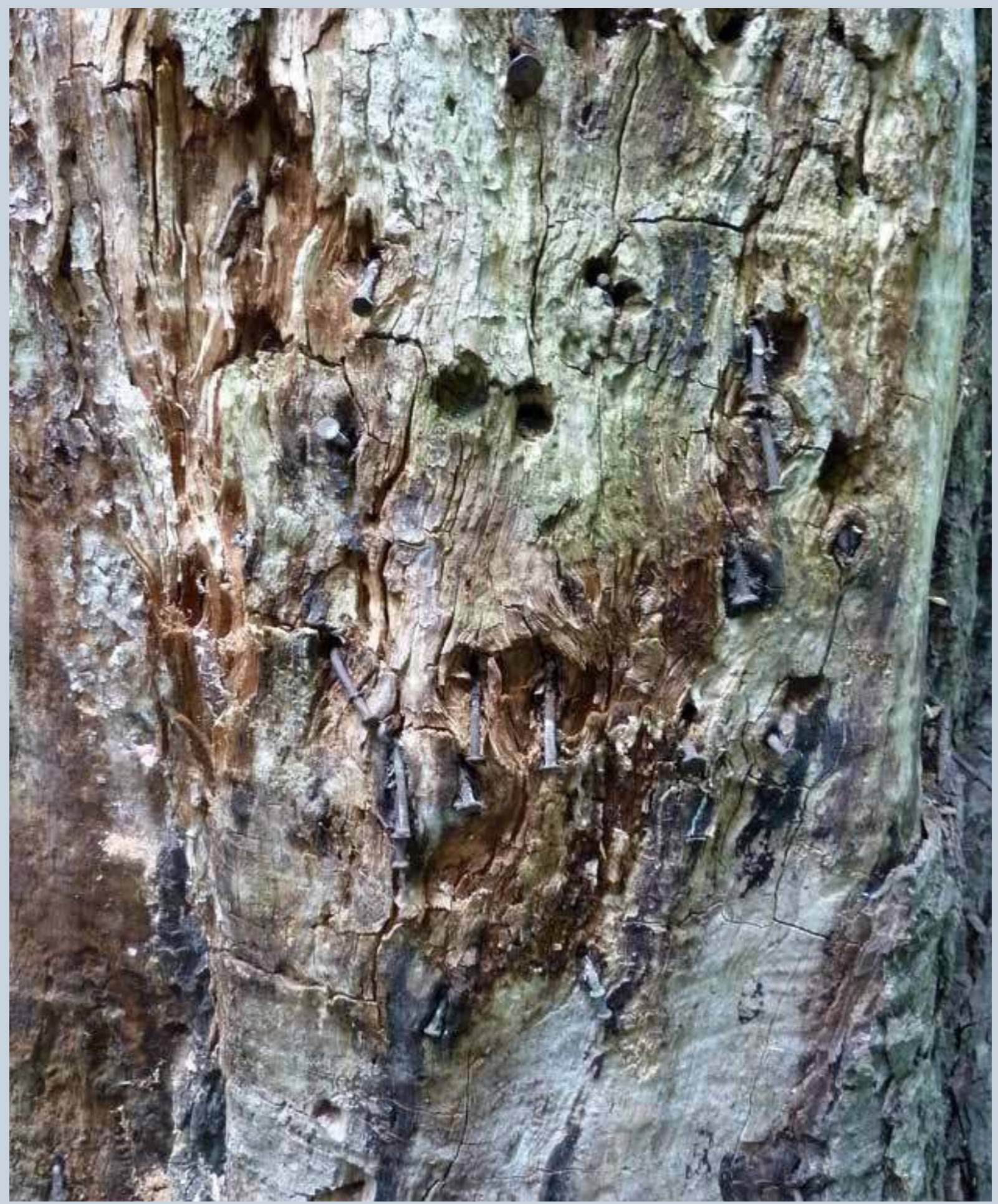




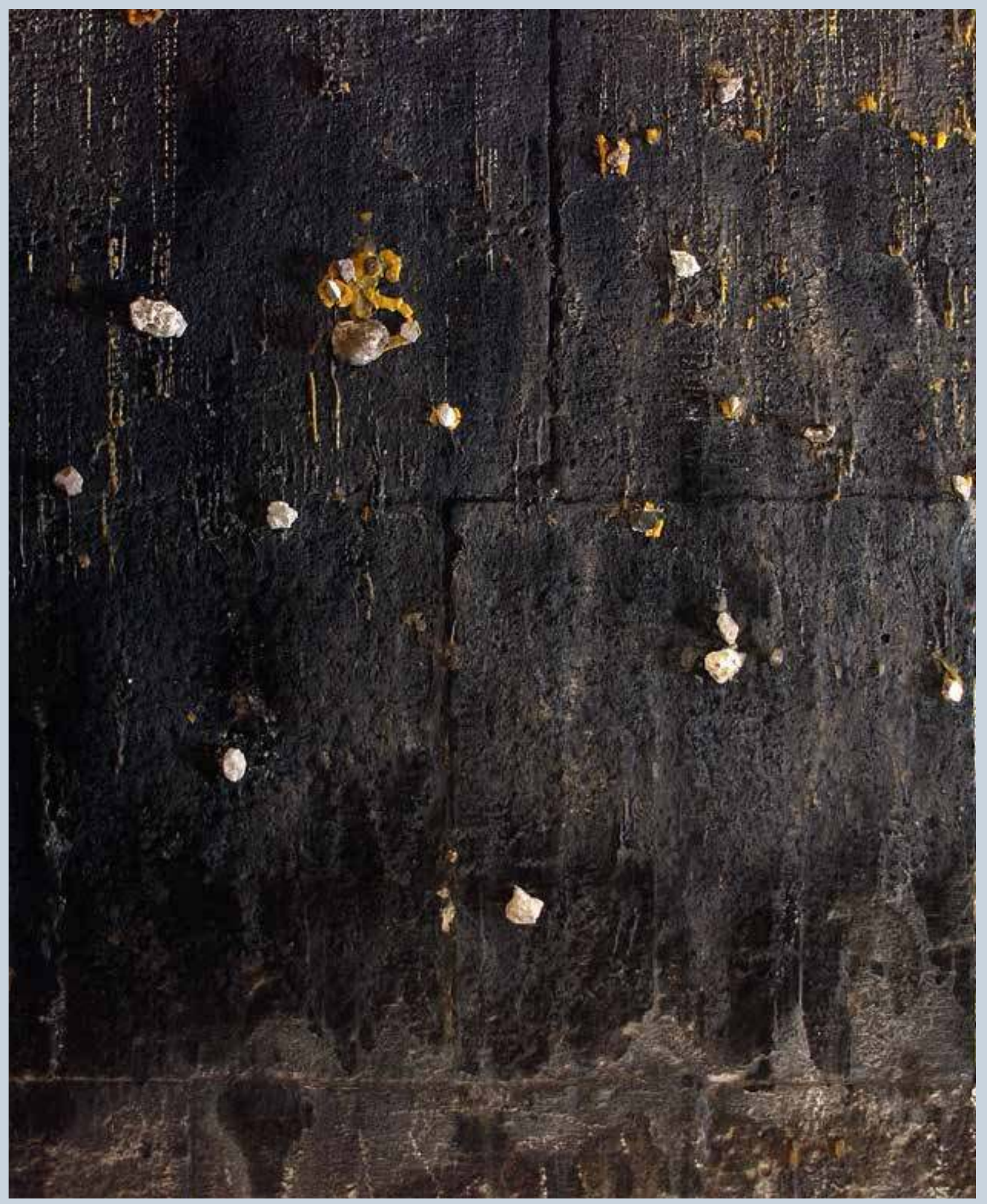




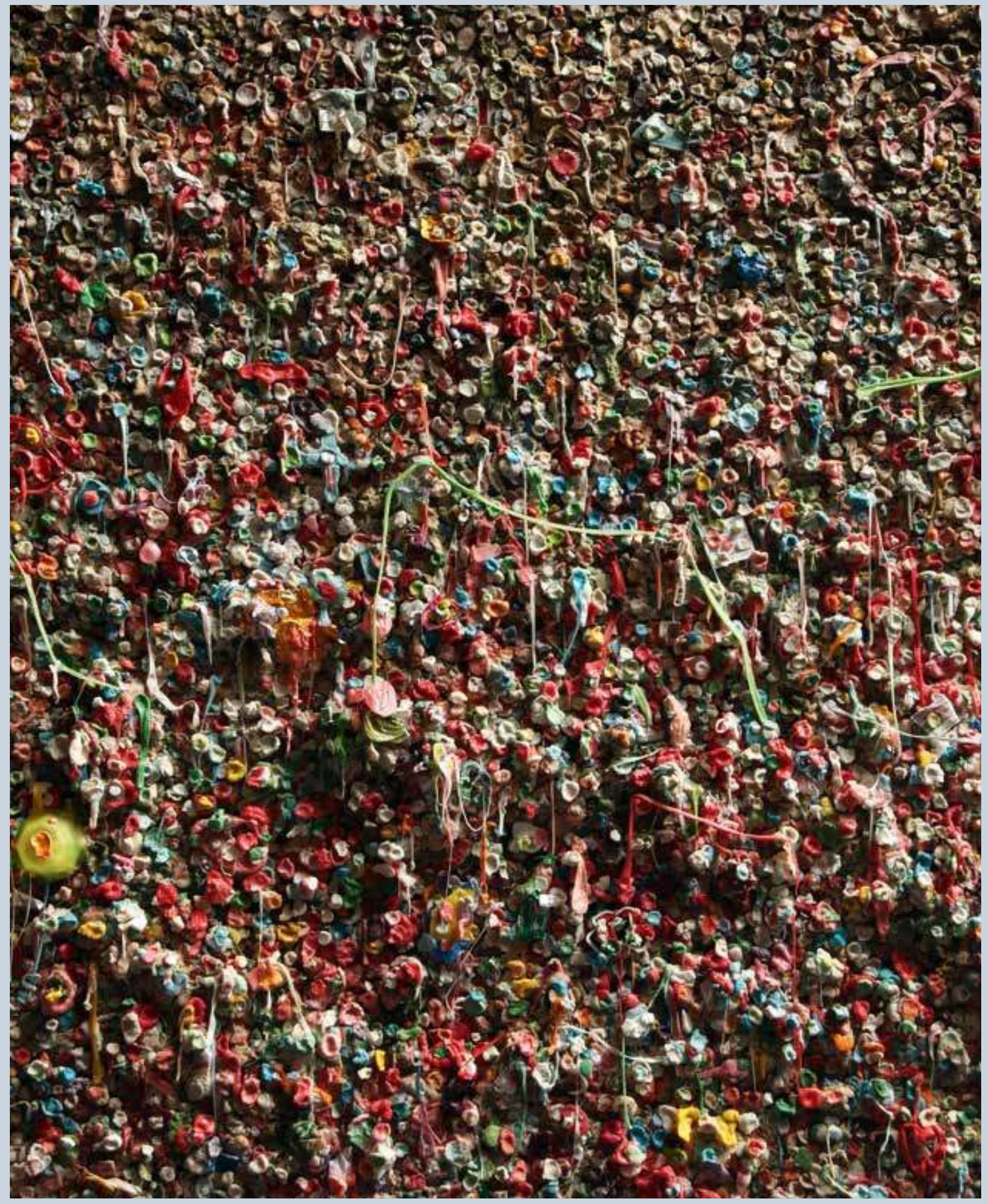




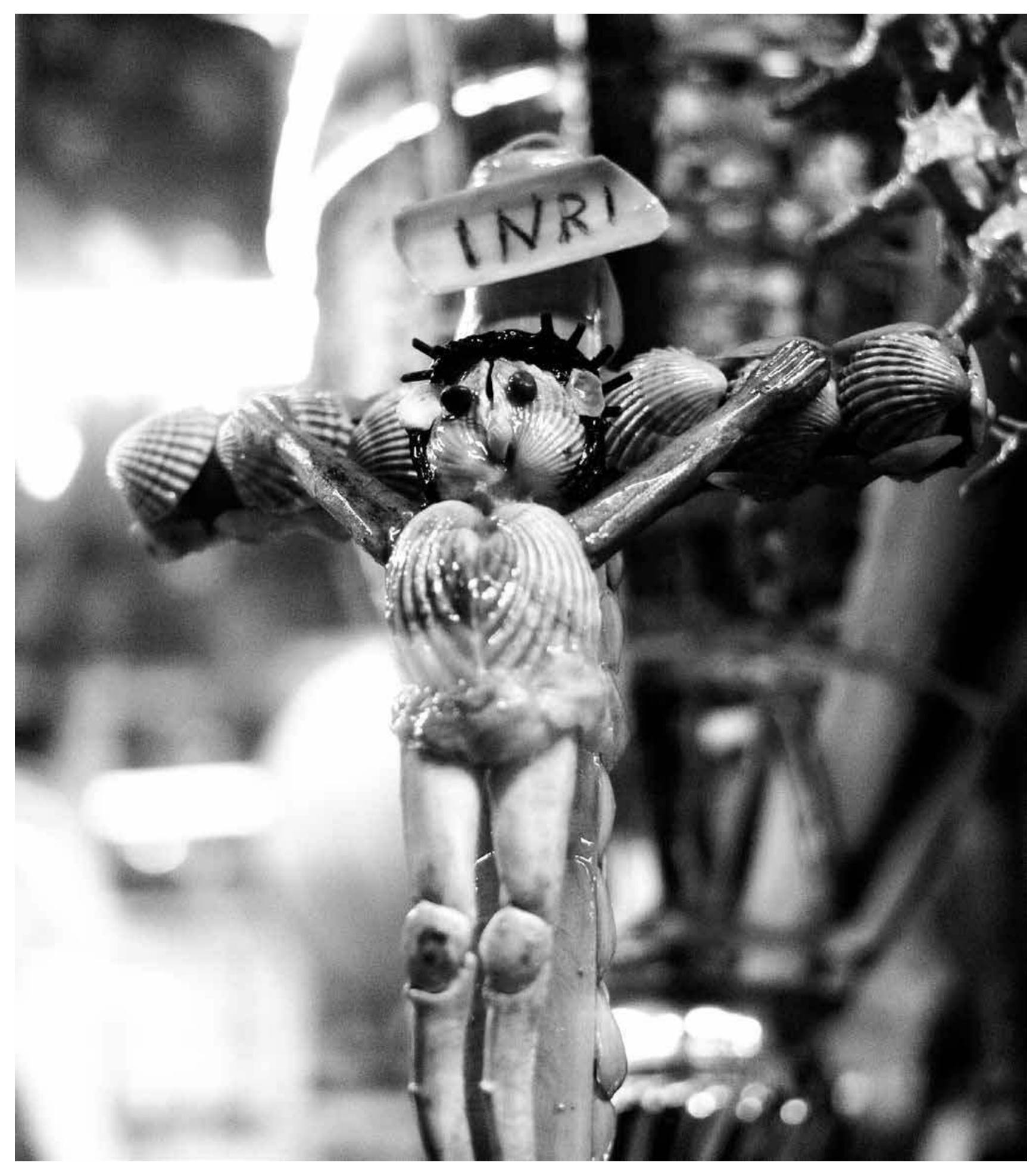


S'il est vrai, comme le dit Testart, que les relations des humains avec les puissances invisibles sont analogues à l'organisation de leur société, on peut concevoir que les offrandes votives aux dieux ressemblent aux pratiques oblatives entre les humains. Il faut imaginer que l'acte de la fidèle du Moyen Âge qui offre un utérus en cire à la Vierge tout en lui demandant de lui octroyer la grâce d'être enceinte, puisse être similaire à ce qu'on voit dans cette scène fameuse du Parrain de Francis Ford Coppola, dans laquelle don Corleone recevant, les uns après les autres, ceux qui désirent bénéficier de sa protection et de son aide, leur demande en échange d'accepter de lui rendre un jour «un service qu'ils ne pourront pas refuser». Cet échange informel, différé dans le temps, qui ne fait l'objet d'aucun contrat, qui ne passe par aucun intermédiaire, qui ne s'accompagne d'aucune transaction financière, qui établit un lien d'attachement entre deux personnes, n'obéit pas à la logique comptable de l'échange marchand: le don divin ne sera jamais remboursé à même hauteur par l'offrande humaine. Il s'agit plutôt d'une logique sacrificielle, au sens large: le don que je promets ou que j'accomplis me prive de quelque chose, et cette privation peut attirer la bienveillance des puissances supérieures: «If friends make gifts, écrit Marshall Sahlins, gifts make friends. » (Sahlins 1972: 186.) Le don aux dieux permet de transformer une relation avec une entité impersonnelle et lointaine en une relation avec une entité puissante mais attentive, protectrice. Il n'y a pas de «magie» du don qui résiderait dans l'esprit de l'objet donné, dans le hau maori tel que le comprenait Mauss; mais simplement une relation de confiance, une alliance établie à travers le don. Le don manifeste cette relation; parfois, il la produit, il la force. Mais alors c'est un pari: il faut que l'autre partie se sente dans une égale confiance pour rendre librement le don. C'est ainsi que deux chefs d'État ou de tribus scellent une alliance par des cadeaux mutuels. L'objet donné est chargé de l'« agentivité » des partenaires, pour parler comme Alfred Gell. Dans le don votif, la même logique opère, mais l'un des deux partenaires est une entité invisible dont on postule le don.

Tout se passe comme si, en ajoutant le don à la demande, le ou la donataire assimilait de facto la relation qu'il ou elle cherche à avoir avec cette entité surnaturelle à une relation qu'il ou elle aurait avec un être humain en position de supériorité bienveillante. La pratique de l'ex-voto indique une conception matérialiste des liens avec l'invisible. Les dieux, esprits et puissances à qui l'on adresse des vœux ou donne des offrandes, ne sont pas pensés et ressentis comme le Dieu de Pascal ou le Dieu de Maître Eckhart, le Dieu silencieux, impossible à saisir, tout-puissant mais infiniment lointain, créateur de toutes choses mais absolument retranché dans son être incommunicable. Au contraire, dans le cas des dieux qui écoutent les prières, reçoivent les dons et éventuellement y répondent, le royaume de l'au-delà n'est pas essentiellement différent du palais du seigneur sous la domination duquel on se trouve; la foi est moins une question de croyance en l'existence d'un Créateur que de confiance envers ces puissances protectrices. Il peut arriver, néanmoins, que les demandes deviennent doléances et que le palais du seigneur soit mis à sac par ses sujets mécontents; ainsi des dévots mexicains, qui peuvent «punir » les images des saints jusqu'à obtenir satisfaction. On touche là au point où la relation au divin devient si matérielle qu'elle s'équilibre et perd toute transcendance.

8. Cristo delle vongole Sanctuaire du Divino Amore
Légendes des figures pages précédentes:

6. Cailloux votifs

Odzun, Arménie

7. Gum wall

Seattle, 2015 


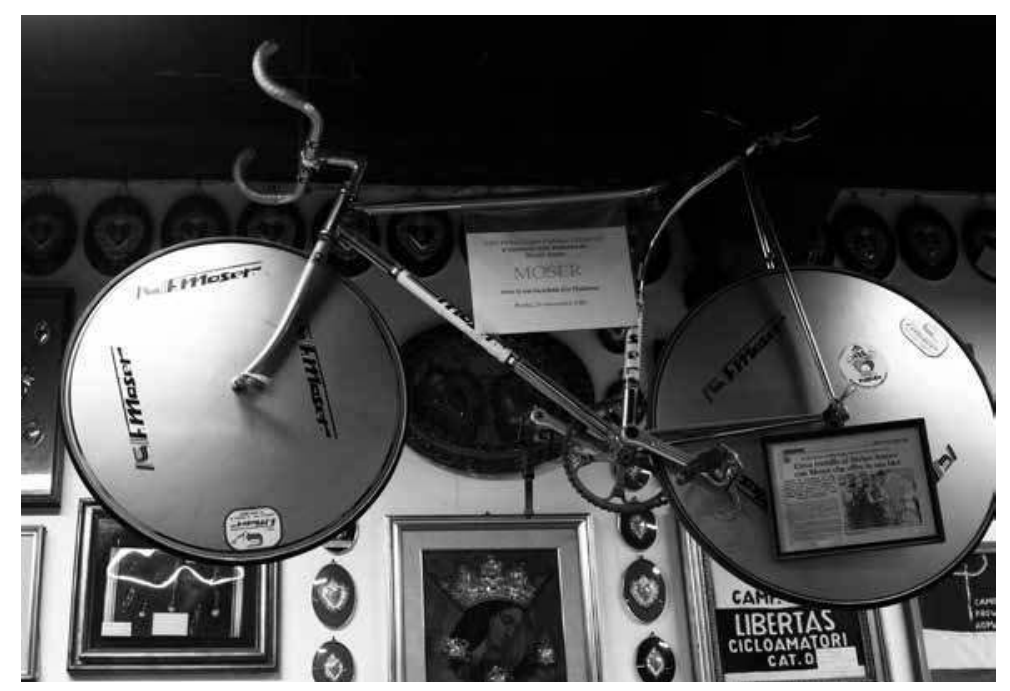

9. La bicyclette de

Moser

Francesco Moser et Eddy Merckx sont tous les deux champions cyclistes, tous les deux se sont reconvertis en créant leur marque de vélo, et tous les deux ont offert leur monture victorieuse à la vierge du Sanctuaire du Divino Amore, dans la banlieue de Rome.
Si le sacrifice est un tribut exigé par les dieux ou les esprits, l'ex-voto est plutôt une reconnaissance de dette; mais dans les deux cas, la perte de la chose donnée est le prix à payer pour établir un lien de sympathie, pour s'attirer les faveurs des puissances supérieures, parfois pour les exiger. La question reste de déterminer comment ce prix s'établit, c'est-à-dire la valeur de l'ex-voto. Jacques Godbout considère que ce qui est donné n'a ni une valeur d'échange (marchande, numéraire) ni une valeur d'usage (utilitaire, de fonctionnement): c'est la «valeur de lien» (Godbout 1992: 244-245), c'est-à-dire «ce que vaut un objet, un service, un "geste" quelconque dans l'univers des liens, dans le renforcement des liens. »

Mais l'ex-voto peut en réalité être évalué de quatre manières: comme objet produit et vendu à quelqu'un, il possède une valeur d'échange $(\mathrm{VEc})$; comme instrument consommé au service d'une promesse ou d'un vœu, ou d'un acte politique (comme manifestation de puissance par exemple), il a une valeur d'usage (VU); comme don, c'est sa valeur de lien qui compte (VL); enfin, comme objet, il possède une valeur d'exposition (VEx). Ainsi un fidèle chrétien peut-il payer un cierge en cire pour quatre sous ou beaucoup plus aux fabricants (VEc), avant de l'offrir à la Vierge ou au saint local (VL) afin de le ou la remercier de la guérison de sa maladie (VU). Le cierge brûle au milieu de dizaines d'autres jusqu'à ce que le clergé décide de le recycler (VEx). Une bicyclette, achetée chez un vendeur d'occasion de Mexico (VEcl), peut être offerte à la Vierge du sanctuaire de San Juan de Los Lagos (VL), avant d'être revendue dans le bazar adjacent par les administrateurs du lieu (VEc2), pour reprendre un exemple développé plus loin par Caroline Perrée. Le propriétaire de la bicyclette s'en est servi pour faire son pèlerinage (VU1), puis il l'a offerte à la Vierge comme preuve de sa venue en pèlerinage, conformément à la promesse qu'il avait faite (VU2). La différence de ces deux exemples est que le cierge a été spécialement fabriqué pour servir d'offrande votive: c'est une offrande par destination, tandis que la bicyclette a vu sa valeur d'usage évoluer: c'est une offrande par transformation, selon la fameuse distinction discutée ici par Élisabeth Goussard. Autre exemple intéressant : les coin trees étudiés par Ceri Houlbrook attestent de la transformation de l'objet associé par excellence à une valeur d'échange, la pièce de monnaie, en don votif; les pièces quittent ainsi le domaine de l'échange marchand et assument une nouvelle valeur d'usage (elles servent à aider à la réalisation d'un vœu), en recevant une nouvelle valeur, la valeur du lien qu'elles permettent d'établir entre le donateur ou la donatrice et la puissance sacrée de l'arbre. 
Existe-t-il une solidarité mesurable entre ces valeurs dans l'ex-voto? Un objet qui coûte cher, à forte valeur d'échange, possède-t-il une grande valeur d'usage et une grande valeur de lien? Contrairement à ce que pourrait suggérer l'évidence de la logique marchande, il n'existe pas de relation proportionnelle mécanique entre les valeurs de l'ex-voto. L'offrande d'un ex-voto n'est pas assimilable à l'achat d'un service, comme pourrait le laisser croire une certaine pensée utilitariste marquée par le capitalisme. L'acte de donation s'accompagne en effet généralement de toute une série de gestes rituels, de prières, de demandes, de productions d'affects qui ne sont pas quantifiables. Un

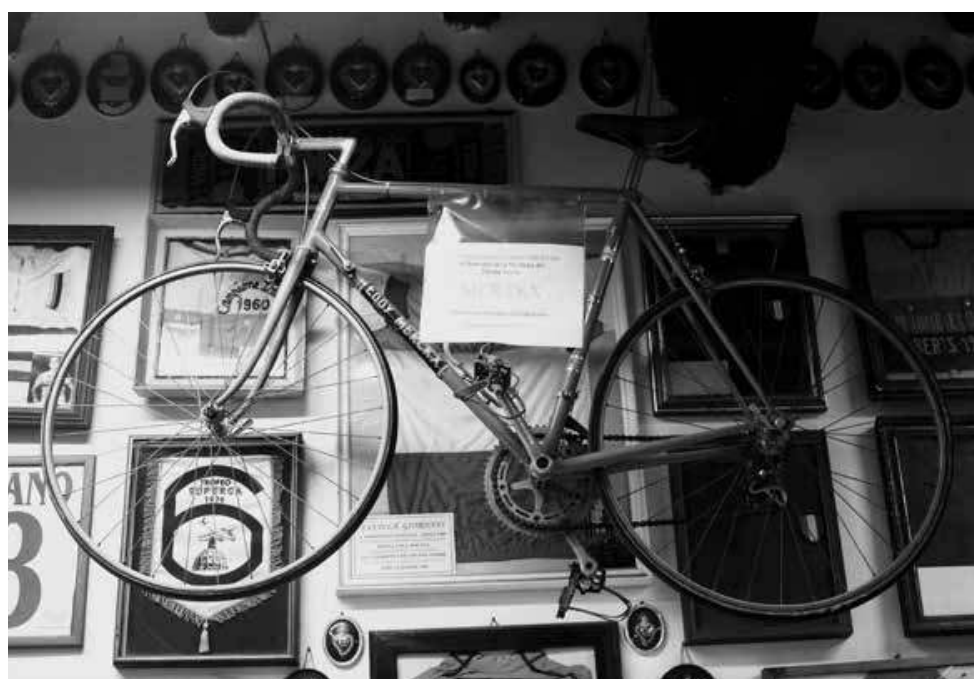
grand pécheur du xiIle siècle peut décider de s'offrir lui-même en ex-voto et de donner à Dieu un an de sa vie, par exemple en accomplissant un pèlerinage à Jérusalem: la valeur de lien est ici énorme, mais comment faire pour évaluer 10. La bicyclette de Merckx la valeur d'échange? On peut aussi offrir un objet d'un coût infime ou nul mais doté d'une forte valeur affective (c'est-à-dire d'une valeur de lien) très forte, comme des effets personnels: le sanctuaire du Divino Amore à Rome possède une salle entière où, de l'aveu même de la sœur qui en assure la surveillance, les fidèles donnent «ce qu'ils ont de plus cher». Le lieu regroupe une incroyable collection d'« objets irremplaçables» (voir Techniques\&Culture 58 [2012]): des souvenirs d'enfances, le T-Shirt avec lequel un fidèle a passé le bac, ou celui du footballeur Francesco Totti lors d'une victoire historique, la bicyclette d'Eddy Merckx, etc. L'attachement affectif à un objet rend ici sa valeur incommensurable, empêche son usage marchand mais en fait un don idéal. Dans le même lieu se rencontre enfin une série d'objets réalisés par les fidèles eux-mêmes, où, comme dans le Japon contemporain, on peut peindre soi-même une simple tablette de bois et remercier ainsi la divinité d'avoir échappé à la mort. Entre art brut et readymade, ces créations mettent en scène les paradoxes de la valeur en contexte votif, jouant sur le contraste entre la grande simplicité des matériaux, et l'incroyable investissement personnel dont ces objets, œufs peints, Christ en coquillages, témoignent.

À l'offrande votive peut s'appliquer la leçon de Théophraste: le philosophe grec estime qu'il vaut mieux sacrifier souvent que sacrifier richement, car dans le premier cas c'est un signe de religiosité, dans le second un signe de richesse (Veyne 1976: 495). La variante proposée par Jésus de Nazareth est intéressante: voyant qu'une femme pauvre mettait deux piécettes dans le Trésor du Temple, alors que des riches mettaient beaucoup d'argent, il dit à ses disciples: «En vérité, je vous le dis, cette veuve, qui est pauvre, a mis plus que tous ceux qui mettent dans le Trésor. Car tous ont mis de leur superflu, mais elle, de son indigence, a mis tout ce qu'elle 
possédait, tout ce qu'elle avait pour vivre.» (Marc XII 43-44.) La valeur de lien d'un don votif ne se mesure pas tant à la valeur d'échange qu'il possède dans le commerce, mais à la privation qu'il représente pour le ou la donataire. Pour des paysans d'Italie du sud ou du Mexique, accomplir un lointain pèlerinage et dépenser quarante euros pour un ex-voto en argent ou en espèces sonnantes et trébuchantes peut représenter un sacrifice important.

Mais, parfois, la valeur d'échange et la valeur d'usage, si ce n'est de lien, de l'ex-voto sont associées, comme dans le cas de l'ex-voto communal d'Ascoli Piceno, ou dans la Madone de Foligno peinte par Raphaël, ex-voto commandé par Sigismondo de' Conti en 1511 pour remercier la Vierge d'avoir protégé sa maison frappée par la foudre. Dans ce cas, comme dans la plupart des autres dans lesquels le don votif possède une valeur d'échange importante, le donataire occupe une place prééminente dans la société. De plus, sa valeur d'exposition augmente alors. «Donner, c'est donner à voir», écrit Testart (ibid: 98) : l'ex-voto possède toujours une valeur d'exposition puisqu'il est la matérialisation visible d'un désir. Mais un ex-voto de papier, un bâton d'encens qui brûle, n'a pas la même valeur d'exposition qu'un ex-voto d'argent, un mannequin de cire ou le tableau d'un peintre prestigieux. On a déjà souligné les limites du jugement esthétique pour qualifier les ex-voto, qui pour cette raison ont longtemps été exclus de l'histoire de l'art. Mais il ne faut pas nier non plus la puissance visuelle que possèdent certains, ou que confère leur agencement ornemental. De nombreux ex-voto sont des bijoux et des vêtements, ornements du lieu cultuel ou de l'effigie sacrée.

Les cadenas d'amour du Pont Neuf, pour la plupart d'entre eux, ne sont pas pour rien dorés ou chromés. Les milliers de bâtons d'encens qui brûlent dans la cour du sanctuaire du Pic du Sud, en Chine, étudiés plus loin par Georges Favraud, produisent un effet pyrotechnique impressionnant. Dieux et humains se réjouissent sans doute les uns et les autres de ces spectacles. C'est par leur impact visuel que les ex-voto manifestent la cohésion de la communauté.

Mais qu'il s'agisse d'un don prestigieux offert par un prince pour manifester avec éclat sa piété, ou d'une offrande modeste noyée dans une masse d'offrandes semblables offerte par un quidam, l'ex-voto est attaché à la personne qui le donne; sa valeur d'échange ne détermine ni son efficacité (valeur d'usage) ni l'obligation de retour (valeur de lien). L'ex-voto n'est pas une marchandise: c'est une métonymie. Le don transforme l'objet en personne ${ }^{8}$. En ce sens, les pratiques de dons votifs qui se sont mondialisées et diversifiées depuis une vingtaine d'années manifestent une certaine résistance à l'ultracapitalisme contemporain qui a tendance, au contraire, à réduire tout être vivant à l'état d'objet commercialisable.

\section{Temporalités : se fondre et se singulariser}

Chaque objet votif produit un rapport au temps singulier, qui invite à s'interroger sur l'intention des acteurs. Les marins naufragés - pour revenir brièvement à cet exemple «canonique» que l'on a ici délibérément laissé au magasin des antiquités ${ }^{9}$ - ont-ils toujours fait un vœu avant 
de déposer un tableau votif? Certes, ils ont échappé à la mort par ce qu'ils considèrent alors, la cause étant désespérée (et les petits tableaux qui furent innombrables au xixe siècle nous montrent souvent les hommes d'équipage dans un état de passivité, presque extatique dans l'attente d'un fin certaine), comme le fruit d'une intervention divine. Mais ont-ils eu l'intention de faire ce dépôt? Plus généralement, peut-on appeler ex-voto ce qui ne résulte pas d'un vœu antérieur? C'est une question passionnante et difficile, car elle pose le problème de tous les actes qui ne sont pas précédés d'un vœu mais qui accompagnent un vœu: deux temporalités ici, qui ne se confondent pas, ou que, tout au moins, il importe méthodologiquement de distinguer pour mieux pouvoir, ensuite, en montrer les imbrications. Dans le premier cas, l'objet atteste d'un vœu antérieur, dans le second il témoigne d'un vœu présent. Mais comment pouvons-nous savoir ce qu'il en est de ce vœu antérieur autrement que par l'attestation qui en est produite? Et, inversement, le vœu immédiat, projeté sur un temps à venir, ne procède-t-il pas obscurément d'une première intention? Nous retrouvons-nous jamais par hasard dans un sanctuaire? Le petit mot d'ex-voto est précieux, parce qu'il maintient ouverte la possibilité de cette antériorité.

Par ailleurs, plus généralement, l'accomplissement d'un vœu passé n'est-il pas aussi une promesse? Ne fait-on que s'acquitter d'un vœu? Ou contracte-t-on toujours une nouvelle dette, qui est aussi une nouvelle assurance? Comment un vœu ponctue-t-il le temps d'une vie, en le rythmant sans le borner?

Par-delà la question de l'intention, les objets eux-mêmes témoignent de rapports au temps particulièrement contrastés. Dans le foisonnement des cas et des situations, deux types semblent se dégager, qui sont aussi deux rapports différents au collectif.

Il faut d'abord considérer les dépôts votifs qui s'inscrivent dans une vie cyclique. Qu'il s'agisse

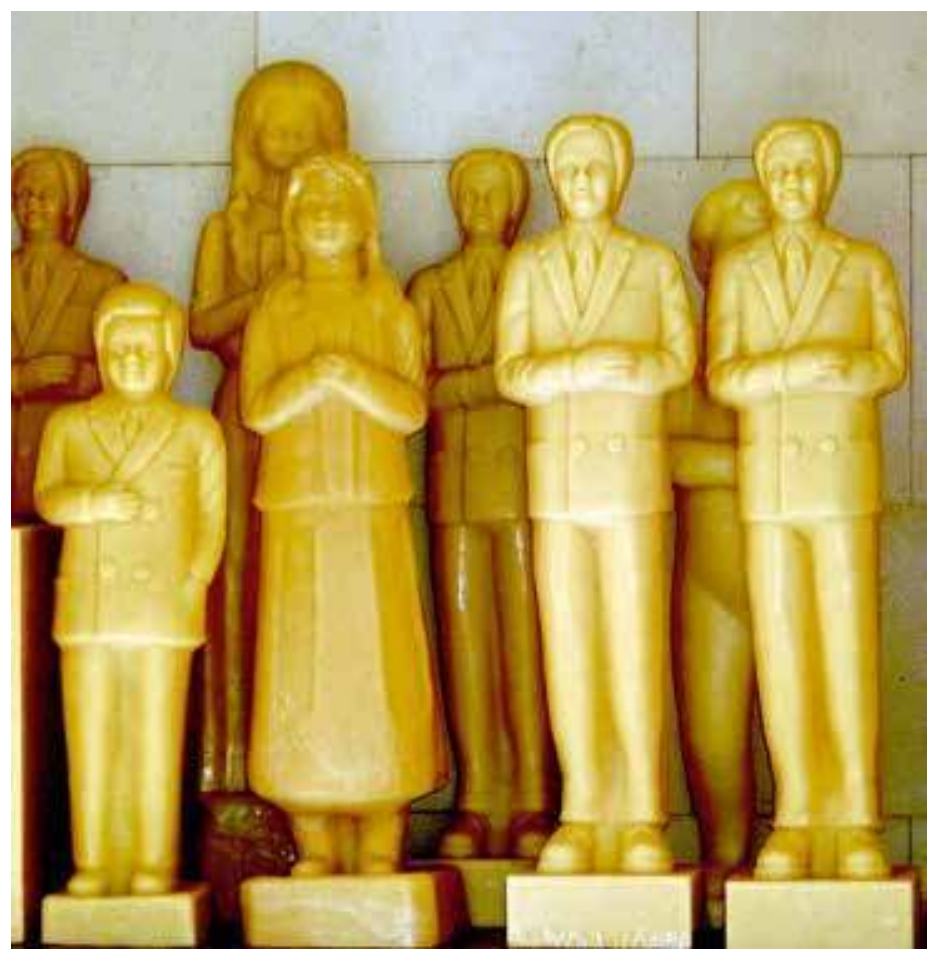
d'ex-voto anatomiques en cire ou en métal, de simples bougies, ou de mottes de cire, nous sommes face à des objets sériels produits dans un cadre industriel ou proto industriel, qui impliquent une anonymisation des corps et des désirs. Objets pauvres, standardisés, mille fois moulés et démoulés, ces dons s'inscrivent généralement dans une temporalité rapide: achetés sur les lieux mêmes des pèlerinages, leur temps de vie est largement encadré par des institutions qui gèrent les temps d'exposition, installent ou enlèvent ces objets en fonction des flux, assumant ainsi les modalités pratiques de la médiation avec la 


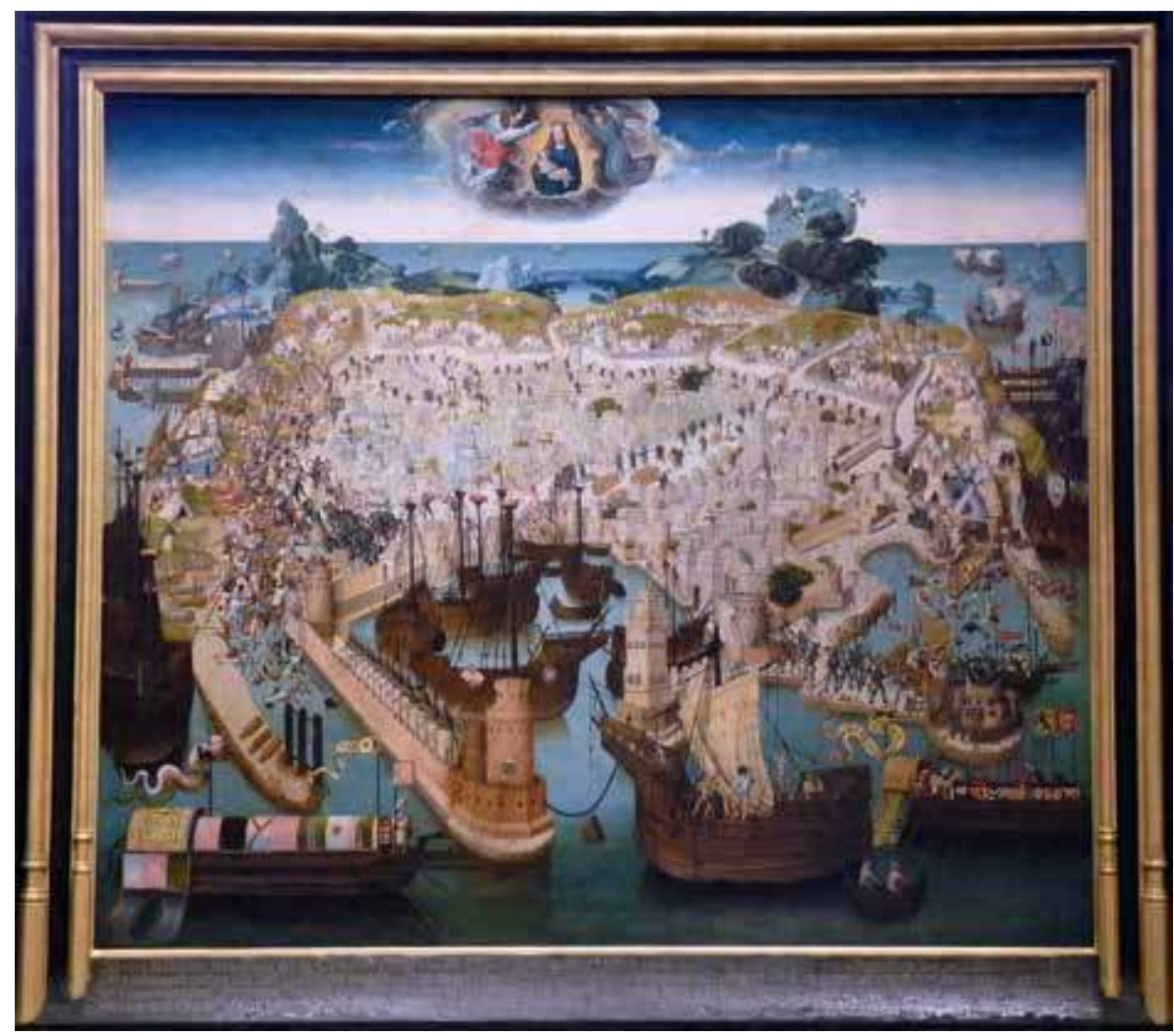

divinité. Le caractère périssable ou recyclable des matériaux employés est ici fondamental: la vie de ces objets est liée à une souffrance ou à un désir que l'on veut penser comme temporaire. Ces fragments de corps sont faits pour disparaître, ils «vivent et meurent» (Charuty 1992). Ils sont revendus, remis en circulation et parfois fondus pour pouvoir accueillir, sous une forme nouvelle, de nouveaux désirs. Anonymes et collectifs, ces corps morcelés possèdent aussi une historicité implicite, la matière de chaque oreille, chaque pied en cire exposé ayant pu non seulement supporter les peines et les souffrances d'autres individus, mais également appartenir à une autre forme, à un autre organe chargé de peine et de désir. Mais que le dépôt soit

12. Le siège de Rhodes, 1480 Cet ex-voto offert à Notre-Dame de Paris a été refusé par les chanoines (Hamon 2009). furtif, éphémère ou pérenne, il importe que l'objet promis au sanctuaire demeure ne serait-ce qu'un instant au contact ou au plus près de la puissance agissante dans le lieu, car les objets des individus deviennent alors, et seulement alors, ceux de la divinité, comme l'observe Vasiliki Zachari en étudiant les pinakes grecs antiques.

Cette forme de dépôt votif traverse toute l'histoire occidentale et oblige l'historien ou l'anthropologue à concevoir des perspectives de très longue durée, inversement proportionnelles au temps éphémère de ces objets. Elle ne saurait cependant constituer un modèle général, puisqu'il existe de nombreux ex-voto dont le rapport au temps est pratiquement opposé à celui-ci.

De fait, depuis le $\mathrm{xv}^{\mathrm{e}}$ siècle en Occident, on assiste à une multiplication d'objets votifs d'un genre différent qui relèverait d'un vœu mémoire. Tableaux réalisés par les plus grands artistes, performances de peinture, sculptures en marbres ou prouesses d'orfèvrerie conservées dans les trésors des églises, ils représentent avec soin l'apparence physique des commanditaires. Ce sont des objets de valeur, réalisés dans des matériaux précieux, réservant souvent une place pour un écrit, explicatif ou invocatif. Ce sont également des objets de prestige, où l'artiste autant que l'institution est cette fois le principal médiateur. Parfois même, des édifices entiers sont des 
ex-voto édifiés par des communautés ou un seigneur, comme lorsque Francesco I Gonzaga de Mantoue fit construire dans les années 1380 à Curtatone le sanctuaire de S. Maria delle Grazie, que Valeria Motta étudie en détail, pour remercier la Vierge d'avoir libéré la région de la peste. Ces vœux mémoire exhibent des singularités. Explicitement faits pour durer, ils témoignent, dans le temps, d'une situation d'exception où l'originalité d'un individu (le commanditaire) et d'un événement (un miracle) semble se répondre et se renforcer mutuellement. Les habitants de Mantoue ont tous bénéficié de l'arrêt de la peste et ils restent tous les obligés du prince Francesco I. Une valeur d'exposition forte s'ajoute ici, car il importe pour le donateur de montrer son don à ses sujets.

Si ce vœu mémoire, témoin singulier de l'acte dévotionnel d'un individu aisé, apparaît dans un premier temps comme l'apanage d'une élite, capable de commande, ce privilège semble s'atténuer au cours des $\mathrm{XIX}^{\mathrm{e}}$ et $\mathrm{xx}$ e siècles, où l'on assiste à une démocratisation de ce type de don, dont les petits tableaux peints, parfois pour un groupe d'individus, sont une première manifestation.

L'âge d'or des tavolette, qui se situe en Europe entre 1850 et 1950, témoigne d'un nouveau désir de durer. Pour modestes qu'elles soient, ces œuvres se singularisent et s'inscrivent dans le temps. Plus encore, la popularisation des moyens de reproduction mécanique (au premier chef la photographie) aboutit à une hybridation des deux catégories que nous venons de distinguer. Une évolution fondamentale du phénomène votif au $\mathrm{xx}^{\mathrm{e}}$ siècle est bien la multiplication des sanctuaires photographiques, où l'accumulation d'images de soi, de ses proches, souvent accompagnées de quelques mots au verso, témoigne de l'appropriation, par des pèlerins modestes, d'une pratique autrefois réservée à une élite. Mais ne nous trompons pas sur les conceptions de l'individu que véhicule ce phénomène que l'on doit comprendre en tenant compte des dispositifs mis en œuvre: l'intense proximité entre ces photographies témoigne d'une volonté d'inscrire l'exhibition de cas individuels au sein d'une communauté affective.

On est ainsi conduit à inscrire l'ex-voto photographique dans une

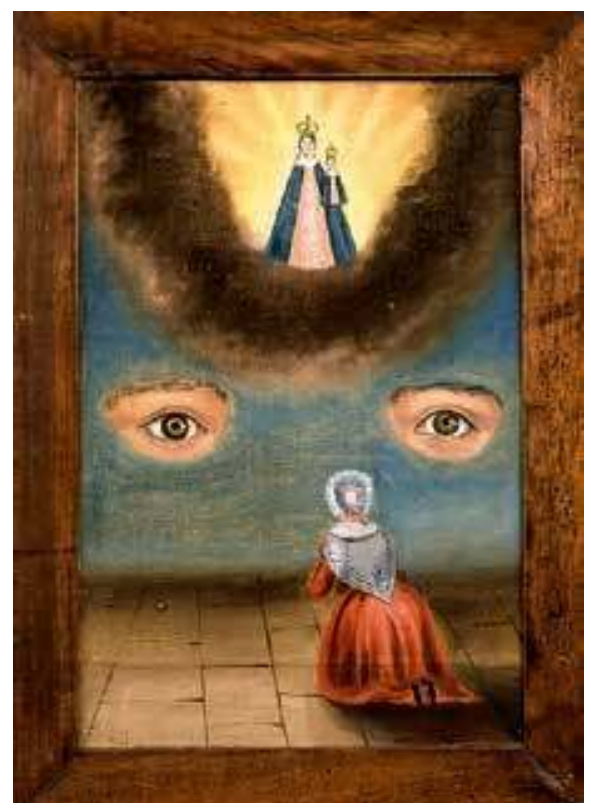
longue durée. On ne peut qu'être frappé, dans de nombreux sanctuaires, par le passage sans transition des petits tableaux peints aux plaques de marbre, et pour une raison simple et forte: il n'y a de représentation de la catastrophe miraculeusement évitée que dans un après-coup. On ne photographie pas l'événement. Et il est vrai que les photographies votives sont très généralement des portraits, d'hommes ou de choses. Mais ne pourrait-on pas imaginer un troisième temps - quelque peu vertigineux - par lequel, dans une sorte d'effet présentiste, on photographierait la catastrophe elle-même, sublime selfie?

Ce troisième temps est déjà d'aujourd'hui : ainsi, au Mexique, au Brésil ou en Italie, la photographie votive fait-elle l'objet d'un véritable travail collectif: l'accident ou l'opération «miraculeuse» sont documentés de l'extérieur, par des photographies de proches, parfois mises en 
série, en collage dans des compositions complexes. Comme autrefois la tablette peinte, l'ex-voto photographique documente ici l'accident et sa résolution.

Ces différents types temporels nous portent à une autre dimension de la question: celle du lieu votif. L'offrande votive doit-elle demeurer dans le sanctuaire pour mériter son nom ou peut-elle ne faire que passer par lui? L'ex-voto peut-il devenir un ex templo? La question de la conservation de l'objet sur le long terme est indirectement posée ici. Si l'on s'accorde à penser que l'ex-voto doit être déposé, peut-il être ensuite légitimement détruit ou recyclé (voire revendu,

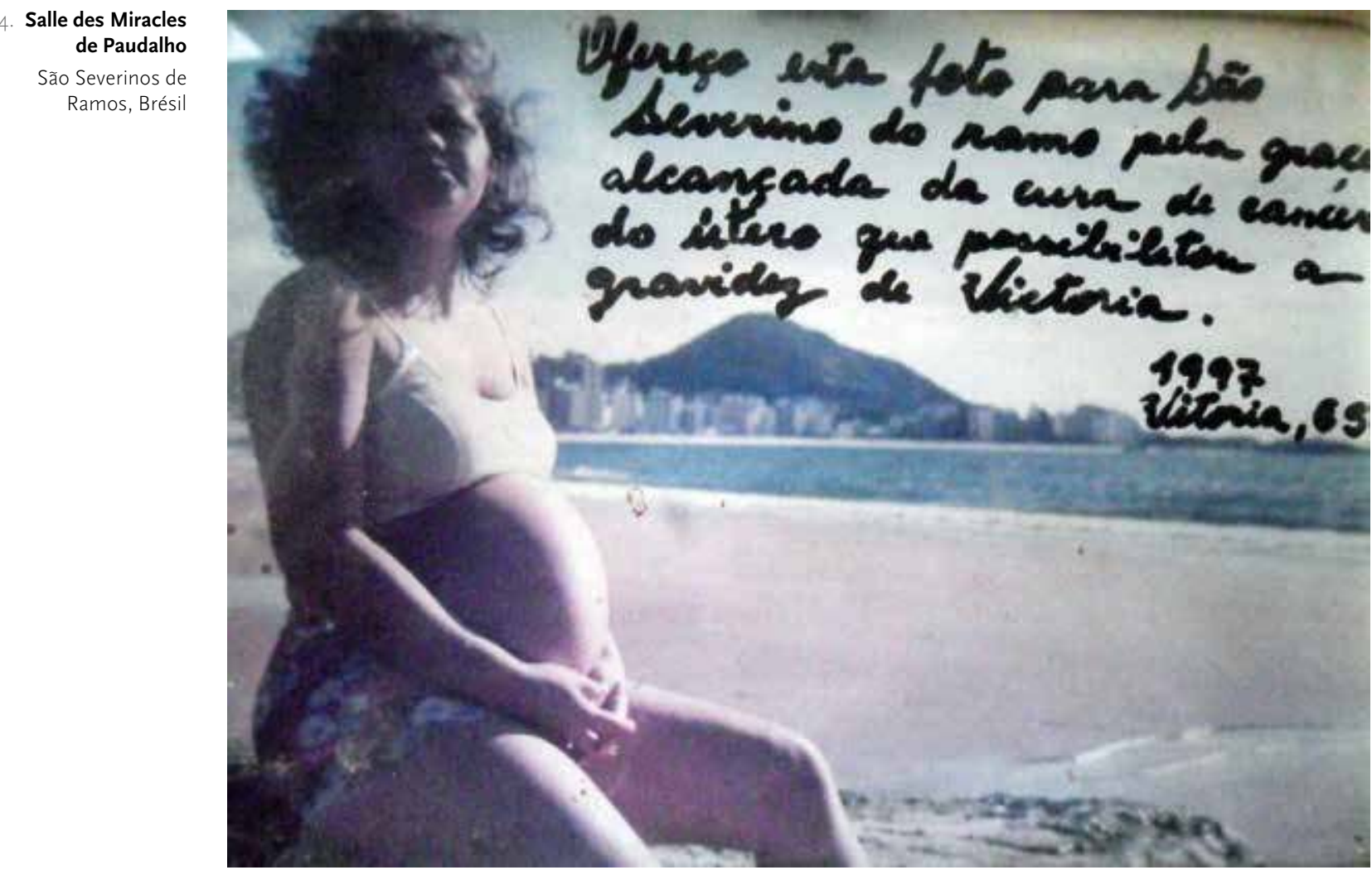

comme on l'observe dans certains sanctuaires mexicains contemporains)? Quel est le centre de gravité de l'acte votif : le geste, ou l'objet? Quelle est, à partir de là, la part de la matérialité dans la médiation votive? Et la part de l'individualité? Car on peut imaginer qu'un objet refondu, redistribué, dé- ou re-qualifié, n'en ait pas moins été efficace, précisément peut-être parce que la singularité de l'acte se confond dans une communauté de foi.

On le voit clairement ici: l'ouverture de l'enquête aux formes les plus apparemment paradoxales ou marginales de l'acte votif, permet d'ébranler une conception sans doute implicitement 
attachée aux modalités les plus répertoriées, images, statues, etc. : le présupposé d'une identité substantielle de l'objet. Peut-être cette conception a-t-elle, de fait, perdu du terrain sur la scène contemporaine, qui nous confronte, par définition, à l'éphémère, à l'événement, à la fugacité des actes. Mais peut-être aussi toutes sortes de formes anciennes ont-elles été oubliées sous leur influence. Aussi est-il essentiel pour nous, avec ce dossier, de tenir une double perspective dans l'espace et dans le temps.

\section{Techniques d'occupation matérialistes}

Les techniques votives sont des techniques collectives. Un ex-voto seul n'a pas de sens, et un don votif n'a de sens que visible par d'autres que soi. Il s'agit non seulement de donner mais dans le même temps, de témoigner et de prendre à témoin. Ne nous y trompons pas, l'expression collective des souffrances et des désirs individuels est toujours aussi une forme de politique, puisqu'elle invoque, voire provoque, des puissances publiques.

C'est moins le message, souvent formulaire et répétitif, qui est revendicatif, que la pratique elle-même qui instaure un rapport de force, parfois au-delà du cadre admis et qui, dans bien des cas, relève de l'occupation des lieux de culte en général, des lieux de pèlerinage, des lieux touristiques aujourd'hui, vers lesquels converge la plus grande population possible - certaines destinations, comme la ville de Rome, rendant difficile la séparation des genres.

Il faut ici considérer les lieux dans le temps pour mesurer l'impact des crises, combien celles-ci peuvent redéfinir la nature des rapports de force entre les institutions et les collectifs désirants. Comment ne pas être frappé par l'accumulation de textes, d'images, d'objets dans les églises pendant la première guerre mondiale, matérialisation accumulée, collective et solidaire d'angoisses toutes singulières? Il faut imaginer Notre-Dame de Fourvière, à Lyon, littéralement envahie d'objets, et la comparer avec son état actuel, où le souvenir de cette occupation ne se présente plus que sous la forme de quelques cœurs alignés par typologie derrière des vitrines. Il faut voir aussi à Mexico, comment peut se construire le culte d'une image, celle de la Guadalupe, sur un mode autoritaire, avec un dispositif de récipients à ex-voto et de tapis roulants empêchant tout arrêt, tout rythme propre, toute appropriation matérielle du lieu par ses occupants qui ne sont plus que des passants.

Qu'il s'agisse d'institutions ecclésiales ou municipales, ces autorités cherchent à prendre le contrôle d'une expression qui les déborde. De Venise à Paris, les cadenas d'amour sont démontés,

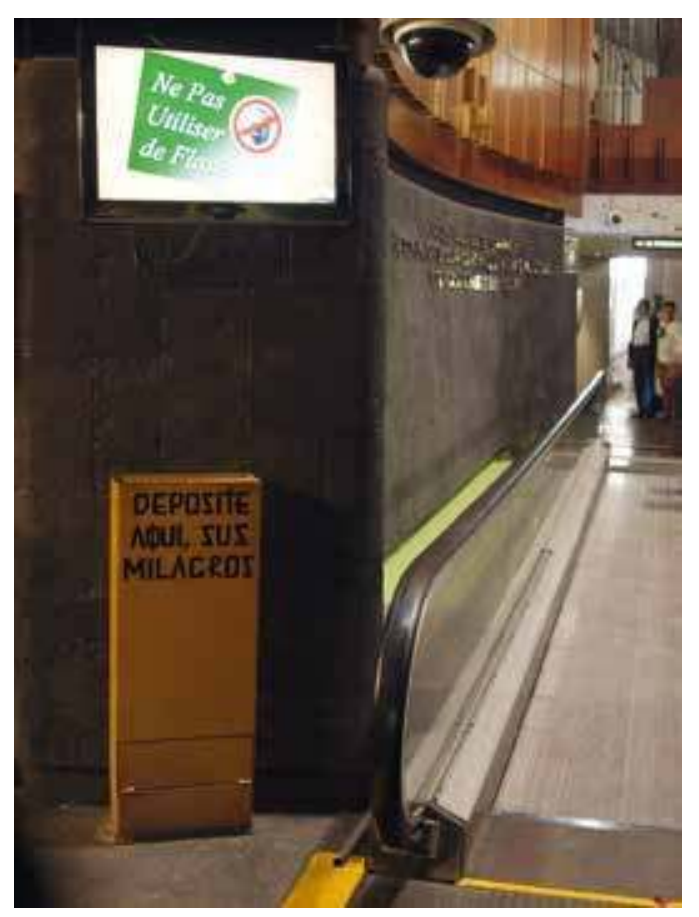
la Guadalupe, Mexico, 2016 
16. Ex-voto de Maria Reyes par David Mecalco, Mexico

«María Reyes remercie la petite Vierge [la Vierge de Guadalupe] d'être une pute et d'avoir de l'argent pour donner à manger à mes enfants. Le 21/01/91. »

Ex-voto de David

Mecalco qui, à l'instar de nombreux créateurs mexicains, invente des peintures à l'iconographie votive mais qui ne répondent pas à une pratique dévotionnelle.

Ces ex-voto souvent subversifs dans leurs thématiques et leurs styles constituent une chronique de la société mexicaine sur un mode

à la fois parodique et réaliste.

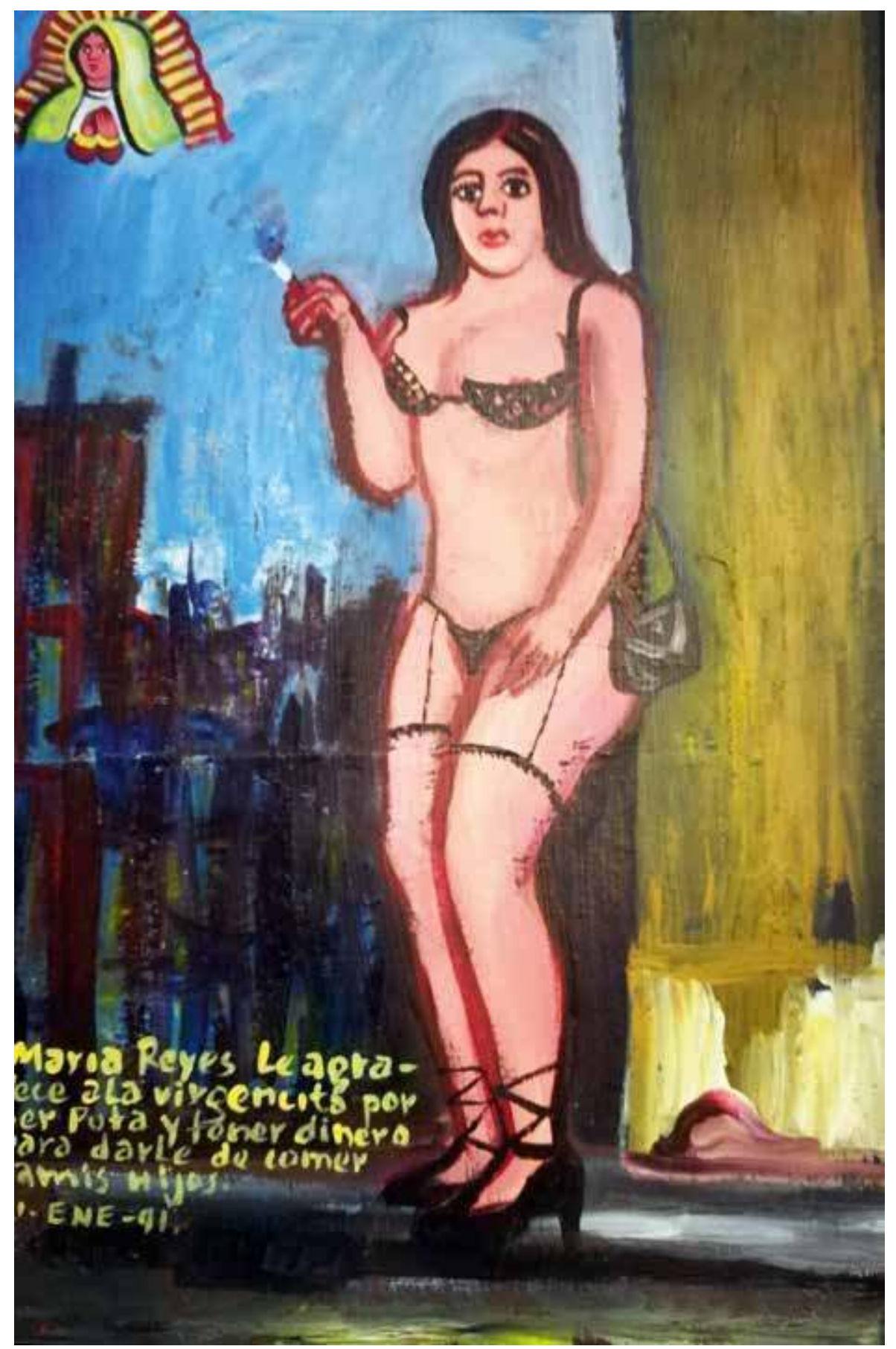


sciés et parfois revendus, les gum walls sont nettoyés comme on efface des graffitis. Victoires éphémères: à peine le Pont des Arts est-il débarrassé que le Pont Neuf se couvre de cadenas. À Mexico, ce sont les alentours du sanctuaire de la Vierge de Guadalupe qui sont le théâtre de danses, dont les costumes attestent d'une créativité débordante aux couleurs chamarrées et dont les accessoires n'ont rien à envier à une installation d'Annette Messager. La matérialité dévotionnelle se porte à même le corps, c'est pourquoi elle ne peut être contrôlée par les autorités, et ce depuis les premiers jours de la Conquête. Le désir d'occuper l'espace public, d'y déployer ses affects, ses souffrances et ses désirs sans les déléguer à des instances régulatrices, semble devenir toujours plus fort dans le contexte de crise de la représentation politique que nous connaissons aujourd'hui.

\section{De la rue au musée : aller-retour}

Certes, le lieu active l'agentivité de l'ex-voto en articulant du singulier sur du collectif, du présent sur du passé - et, dans les limites que nous venons de rappeler, du personnel sur de l'institutionnel. Mais s'il est simplement exposé, sans prière ou tout autre rituel, l'objet votif peut se « décharger», à l'exemple des «mukasari ema» japonais analysés par Agnès Giard qui, après plus de trente ans, sont remisés au musée car ils ne fonctionnent plus, parce qu'ils ne sont plus honorés par personne; mais c'est peut-être aussi parce que leur seule exposition invite les spectateurs à porter sur eux un regard esthétique. Placés dans un lieu muséographique, ils ne sont plus identifiés à des objets dévotionnels actifs, bien que l'on puisse parfois être surpris par le témoignage de certains gardiens de musée, désemparés par la génuflexion orante d'un visiteur toujours très affecté. Les objets votifs du Bazar de San Juan, étudiés par Caroline Perrée, perdent eux aussi toute charge dévotionnelle lorsqu'ils sont mis en vente: changement de valeur, comme on y a insisté plus haut.

Au Mexique, depuis les années 2000, une vogue de peintures reprenant les codes formels de l'ex-voto a vu le jour avec un succès retentissant, au point que son artiste le plus célèbre, Alfredo Vilchis, a été exposé au Louvre lors de l'hommage rendu à J. M. G. Le Clézio en 2011. Les peintures de Vilchis et de David Mecalco ont trouvé leur place dans les collections du Mucem. Quel statut donner à ces objets, qui ont une forme votive sans en avoir l'intention? Leur iconographie reprend celle des ex-voto, mais les miracles sont, eux, peu conventionnels: un supporter célèbre la victoire d'un luchador (un lutteur de catch), la prostituée remercie pour ses clients, la maîtresse remercie San Judas que son mari n'ait pas découvert son amant caché sous le lit, et le retablero, peintre d'ex-voto, implore la Guadalupe de protéger les migrants des méfaits de Donald Trump. Ces ex-voto n'ont jamais été commandés par une tierce personne pour l'offrir au divin et ne trouvent pas leur place dans des sanctuaires, même si Vilchis en a fait quelques-uns sur commande pieuse. Le plus souvent, ces «miracles» sortent tout droit de l'imagination des créateurs, qui n'étaient cependant pas considérés comme des artistes, ni même 
comme des artisans, parce qu'ils vendaient (et vendent encore) leurs productions sur le marché aux Puces de Mexico, la Lagunilla. Leur succès auprès des touristes et collectionneurs n'a fait que les encourager dans cette veine miraculeuse, et ils sont aujourd'hui reconnus comme des artistes et exposés dans des galeries et musées. Alfredo Vilchis est surnommé le Da Vilchis de Mexico en référence à Léonard de Vinci. Si ni leurs récits ni leurs illustrations ne coïncident avec les miracles modelés par la tradition, ces images mettent en scène un Mexique quotidien dans lequel chacun se reconnaît. Exposées ensemble, elles constituent une véritable fresque sociale, sur un mode souvent humoristique comme dans le cas des parodies romaines étudiées par Anne Lepoittevin.

La prise en considération de ces cas limites impose de penser l'artification des formes votives en dehors de l'espace muséal. De fait, les murs d'ex-voto dans la rue constituent aujourd'hui des points d'intensité dans l'espace public, des lieux d'élections qui rentrent en concurrence avec les white cubes du musée ou de la galerie. Le flâneur attentif que nous imaginions au début de cette introduction n'aura pas manqué de remarquer, au milieu des images d'accidents et des demandes d'enfants du Largo Preneste, la présence d'un space invader en mosaïque, œuvre de street art égarée au milieu des vœux «chrétiens». Pour qui s'en donne la peine, il n'y est guère de lieu votif qui ne cache une ou plusieurs propositions artistiques, parfois assumées, parfois discrètes. Surtout, il ne semble plus possible désormais de penser un simple processus partant de dévotions «populaires» pour évoluer vers une muséification stérilisante. L'évolution du mur des offrandes d'Avignon est symptomatique de la complexité des destins contemporains. En 1994, quelques parpaings d'un mur de la prison Sainte-Anne d'Avignon sont éventrés et commencent à accueillir des objets les plus variés. Un artiste, Marq Tardy, revendique depuis cette intervention, pensée au départ comme purement artistique et éphémère. Progressivement l'œuvre échappe à son créateur et suscite des offrandes spontanées, alors généralement interprétées comme étant des dons des familles aux prisonniers reclus derrière ce mur. Mais depuis 2003, date de la fermeture de la prison, le mur n'a eu de cesse de s'enrichir et de se régénérer quand bien même le motif le plus évident d'offrande avait disparu. Menacé de disparition, le mur qui a fait depuis l'objet d'une protection et d'une exposition, se trouve toujours actif aujourd'hui, son renouvellement étant documenté par un groupe d'amateurs en ligne. Une fois de plus, l'importance du dédicataire semble s'effacer au profit des liens horizontaux: la présence d'un micro-évènement urbain dans un lieu signifiant fait signe, et met au travail une communauté d'anonymes. Plus que la relation avec des invisibles, c'est bien une logique de stigmergie qui se met en place: une stigma (une trace) dans la rue, engendre un ergon (une œuvre). Mais cette forme de coopération indirecte ressemble peu aux constructions des fourmis ou des termites qui ont permis de forger ce concept. Nul besoin ici de cohérence, d'aboutir à une quelconque structure systématique, puisque les enjeux sont plus expressifs que constructifs (MacDowall 2015) ${ }^{10}$. 


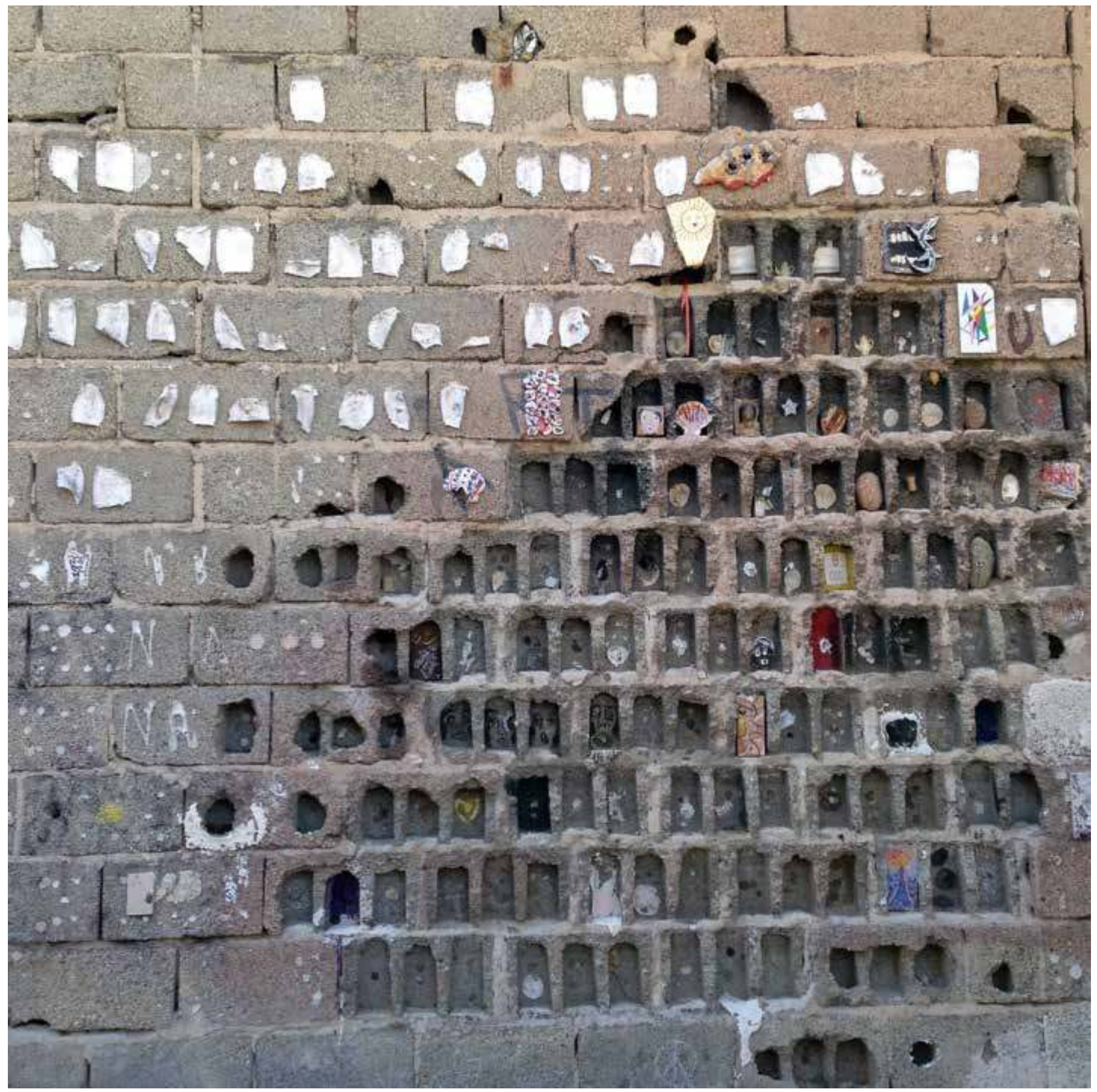




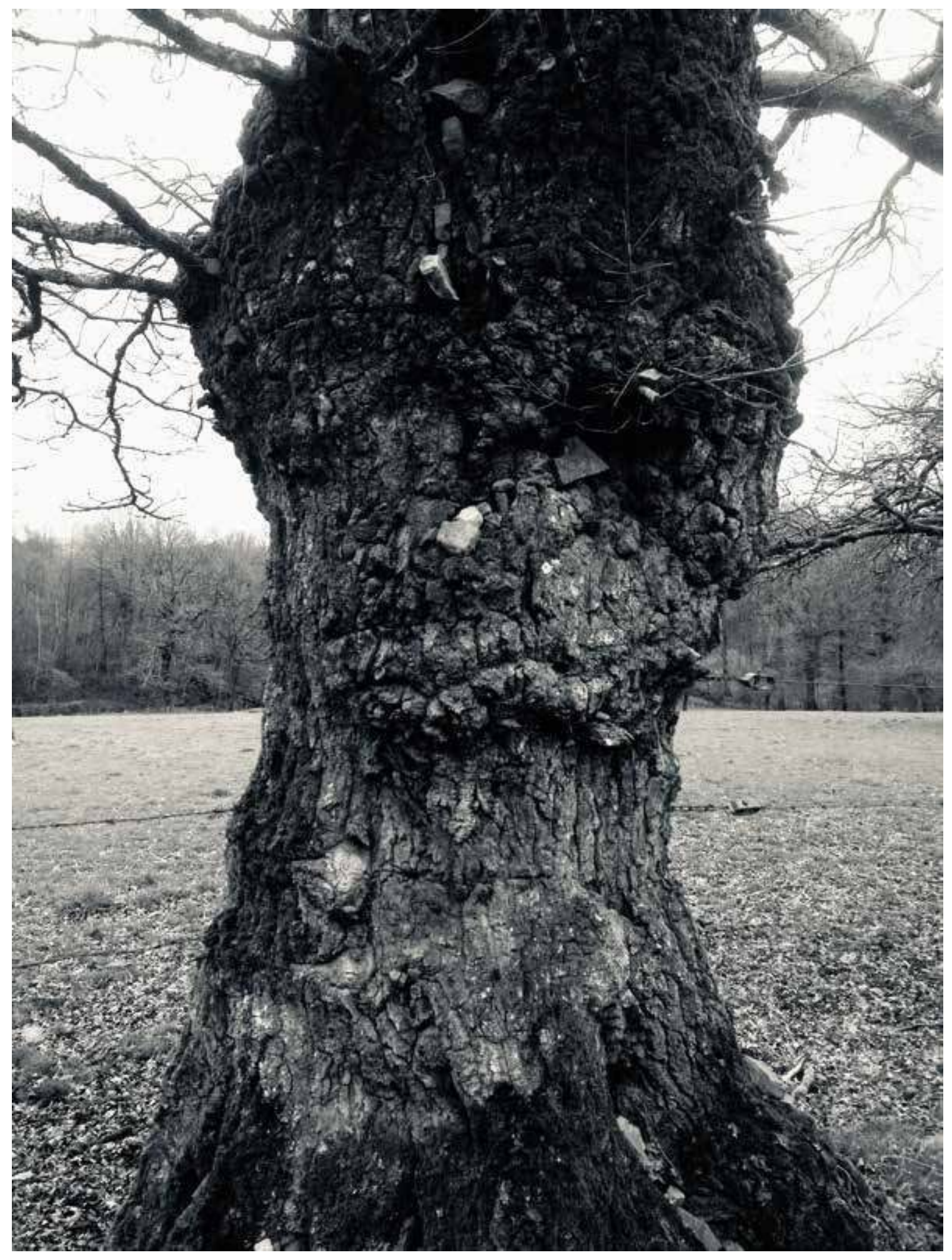




\section{Ouverture : des objets pour faire monde}

Pendant longtemps, les dépôts votifs ont été considérés comme des variantes populaires, des effets secondaires de l'image de culte. C'est à ce titre qu'ils étaient théorisés selon un schéma d'abord vertical, par lequel il s'agissait de comprendre comment le don de choses permettait d'accéder à différentes formes de transcendance. Après avoir exploré dans ce numéro les formes de don les plus contrastées dans le temps et dans l'espace, en prenant en compte, autant que possible, les processus et les dispositifs, le phénomène nous apparaît sous un jour nouveau. L'absence d'une divinité et parfois d'un vœu explicite nous oblige à considérer les dépôts votifs dans toute leur immanence. Dans bien des cas, nous sommes face à des structures horizontales, créant des chaînes d'élection et de solidarités entre ces donataires sans destinataires.

Ces créations collectives indirectes ne se résument pas à une juxtaposition d'égocentrismes. Ces lieux ont désormais largement vocation à tisser des liens et à en rendre compte sur un mode expressif. Ici mieux qu'ailleurs peuvent advenir les relations entre les vivants et les morts, entre les amoureux, entre les membres d'une famille, les liens éprouvés dans les souffrances du travail, de la guerre ou de la misère. Enfin et surtout, on doit comprendre ces communautés créées par ces dépôts comme autant d'interfaces engageant sur un mode affectif non seulement des humains entre eux, mais aussi des individus et des fragments d'un monde qui se donne à voir dans toute son hétérogénéité, avec des voitures démodées, des nattes de cheveux, des chiens, des ânes, des arbres et des cailloux.

18. Cailloux voués à saint Ortaire, Bagnoles 


\section{Notes}

1. «At Diagoras cum Samothracam uenisset, Atheus ille qui dicitur, atque ei quidam amicus: 'Tu, qui deos putas humana neglegere, nonne animaduertis ex tot tabulis pictis, quam multi uotis uim tempestatis effugerint in portumque salui peruenerint?', 'Ita fit', inquit, 'illi enim nusquam picti sunt, qui naufragia fecerunt in marique perierunt.'» (Notre traduction).

2. Ce séminaire a donné lieu à un recueil d'études à paraître dans la revue Pietas, à Munich, et à une journée d'études doctorales coordonnée par Valeria Motta, également en cours de publication.

3. Un «pur amour» dont Jacques Le Brun a admirablement montré les apories.

4. «Item quod ad devotionem perpetuam et in commemorationem Beati Jacobi de Monteprandono fiat fabricari una Gabella in templo Annunziat. expensis Comunis per totum mensem aprilis et fiat unum tabernaculum in dicat Capella (cum tabella picta, barré) de imaginibus gloriose virginis marie ex lapide qui respiciat versus civitatem, cui beatus Jacobus offerat civitatem Asculi cum una lampada que assidue ardeat. Et fundetur honorabiliter accipen. pecuniam undecumque et cogantur omnes muratores ad fabrican. soluta eis competenti mercede. »

5. La section « Le présent fait aux hommes et le présent fait aux dieux » traite essentiellement de différentes formes de sacrifices.

6. Pas d'ex-voto dans M. Sahlins (1976 [1972]), M. Strathern (1988), A. Testart (2006 [1993]), M. Godelier (1996), J. Godbout (2000 [1992]).

7. Ainsi, pour Bernard Cousin, l'ex-voto est un don parallèle à l'invocation au dieu, soit pour l'obliger à intervenir en sa faveur, soit pour désigner de manière figurée l'objet de l'intervention requise, soit pour le remercier du miracle accompli (Cousin 1981 : 11-13). Pour Pierre-André Sigal, l'ex-voto est un objet offert en témoignage d'un miracle ou pour

\section{Iconographie}

Image d'ouverture. Le mur d'ex-voto du Largo Preneste, Rome, 2018. Photographie: Pierre-Olivier Dittmar.

1. London, printed by T. Spilsbury, 1786.

2. (C) Mucem.

3. Photographie: Helle Krog.

4. Photographie: Thomas Golsenne.

5. Arbre de Gravenvoeren, Voeren, Limbourg, Belgique. le provoquer et il insiste sur le caractère conditionnel du vœu : dans un certain nombre de vœux, l'objet n'est donné que si le saint intervient réellement (Sigal 1985: 82). Simone Deyts, dans Images des dieux de la Gaule, définit l'ex-voto comme une offrande pour s'acquitter de son vœu à la divinité et pour la remercier, précisant que le mot s'est étendu à tout don fait à une divinité, avec ou sans inscription (Deyts 1992: 78). Quant à François et Colette Boullet, ils se refusent à voir dans l'ex-voto le paiement d'un service rendu et l'objet d'un marché car «Dieu est libre de ses libéralités » et l'exvoto est « un don gracieux» (Boullet 1978: 12-13). La tendance récente est plutôt à ne pas considérer l'ex-voto comme un don: ainsi Ittai Weynrib (2016: 3) trouve-t-il inopérante la théorie de Mauss qui repose selon lui sur la réciprocité du don et du contre-don, réciprocité impossible à observer, toujours selon lui, dans les pratiques de l'ex-voto.

8. L'ex-voto possède certaines caractéristiques communes avec la relique chrétienne: elle remplace métonymiquement la personne, elle est attachée à un lieu, elle possède des pouvoirs bénéfiques. Mais l'ex-voto est un don fait par les fidèles aux saint.e.s, tandis que les reliques sont, en quelque sorte, les dons des saint.e.s faits aux fidèles.

9. Mais on peut trouver une tentative de nouvelle lecture dans P. A. Fabre et B. Roberjot, «La langue de terre dans les ex-voto marins», in Pietas, op. cit.

10. Le concept de stigmergie est crée en 1959 par PierrePaul Grassé pour décrire le procédé de fabrication des habitats animaux, comme les enveloppes de certains guêpiers, les rayons de cire des abeilles ou les nids de termites. Il est utilisé dans le contexte du street art par L. MacDowall.

6. Photographie: Pierre-Olivier Dittmar.

7. Photographie: Daniel Schwen/Wikimedia Commons.

8 à 10. Photographie: Pierre-Olivier Dittmar.

11. Photographie: Darwinius/Wikimedia Commons.

12. Photographie: Étienne Hamon.

13. C Région Alsace - Service de l'Inventaire et du Patrimoine. 
$14 \& 15$. D.R.

16. Avec l'aimable autorisation de Valérie Perrée Le Rol

17. Photographie: Pierre-Olivier Dittmar.

\section{Références}

Cicéron 45-44 av. J.-C. De natura deorum.

Boullet F. \& C. 1978 Ex-voto marins. Genève: Éditions Maritimes et d'Outre-Mer

Charuty, G. 1992 «Le vœu de vivre», Terrain 18 « Le corps en morceaux» : 46-60. DOI : 10.4000/terrain.3031.

Cousin, B. 1981 Ex-voto de Provence, images de la religion populaire et de la vie d'autrefois. Paris: Desclée de Brouwer.

Deyts, S. 1985 Le Sanctuaire des sources de la Seine. Dijon: Exposition du musée archéologique.

Didi-Huberman, G. 2006 Ex-Voto. Image, organe, temps. Paris: Bayard.

Fabiani, G. 1950 Ascoli nel Quattrocento. Ascoli Piceno: Società tipolitografica editrice vol. I : 167.

Godbout, J. 2000 [1992] L'esprit du don. Nouvelle édition avec la collab. d'A. Caillé, Paris: La Découverte.

Godelier, M. 1996 L'énigme du don. Paris: Fayard.

Hamon, É. 2009 «Un présent royal indésirable : l'ex-voto de la victoire de Rhodes en 1480 à Notre-Dame de Paris », Bulletin Monumental, tome 167 (4): 331-336.

Le Brun, J. 2002 Le Pur amour. De Platon à Lacan. Paris: Seuil.
18. Photographie: Marie-Anne Polo de Beaulieu.

MacDowall, L. 2015 «Graffiti, street art and theories of stigmergy » in J. Lossau \& Q. Stevens dir. The Uses of Art in Public Spaces. London: Routledge: 33-48.

Mauss, M. 1950 [1923-24] «Essai sur le don. Forme et raison de l'échange dans les sociétés archaïques», Sociologie et anthropologie. Paris: PUF «Quadrige»: 164-169.

Revolon, S., Lemonnier, P. \& M. Bailly 2012 «Objets irremplaçables», TechniquesE Culture 58. [En ligne] : journals.openedition.org/tc/6209.

Sahlins, M. 1976 [1972] Âge de pierre, âge d'abondance. Paris: Gallimard.

Sigal, P. A. 1985 L'Homme et le miracle dans la France médiévale (XIe-XII siècles). Paris: Éditions du Cerf.

Strathern, M. 1988 The Gender of the Gift: Problems with Women and Problems with Society in Melanesia. University of California Press.

Testart, A. 2006 [1993] Des dons et des dieux, nouvelle édition. Paris: Errance.

Veyne, P. 1995 [1976] Le pain et le cirque. Paris: Seuil: 495

Weinryb, I. 2016 Ex-voto: Votive Giving Across Cultures. New York : Bard Graduate Center

\section{Pour citer l'article}

Dittmar, P.-O., Fabre, P. A., Golsenne, T. \& C. Perrée 2018 «Un matérialisme affectif», TechniquesE Culture 70 « Matérialiser les désirs. Techniques votives», p. 12-41. 